\title{
Real-Time Estimation of Lane-based Queue Lengths at Isolated Signalized Junctions
}

\author{
Seunghyeon Lee, S.C. Wong, Y.C. Li \\ Department of Civil Engineering, \\ The University of Hong Kong, Pokfulam Road, Hong Kong, China
}

\begin{abstract}
In this study, we develop a real-time estimation approach for lane-based queue lengths. Our aim is to determine the numbers of queued vehicles in each lane, based on detector information at isolated signalized junctions. The challenges involved in this task are to identify whether there is a residual queue at the start time of each cycle and to determine the proportions of lane-to-lane traffic volumes in each lane. Discriminant models are developed based on time occupancy rates and impulse memories, as calculated by the detector and signal information from a set of upstream and downstream detectors. To determine the proportions of total traffic volume in each lane, the downstream arrivals for each cycle are estimated by using the Kalman filter, which is based on upstream arrivals and downstream discharges collected during the previous cycle. Both the computer simulations and the case study of real-world traffic show that the proposed method is robust and accurate for the estimation of lane-based queue lengths in real time under a wide range of traffic conditions. Calibrated discriminant models play a significant role in determining whether there are residual queued vehicles in each lane at the start time of each cycle. In addition, downstream arrivals estimated by the Kalman filter enhance the accuracy of the estimates by minimizing any error terms caused by lane-changing behavior.
\end{abstract}

Keywords: isolated signalized junction, discriminant model, Kalman filter, conservation equation, queue-length estimation.

\section{INTRODUCTION}

Adaptive traffic control systems (ATCS) traffic management strategies in which traffic signal settings are based on the actual traffic demand of the network. To construct the adaptive signal control logic for a real-time signal plan, an estimation of queue lengths is needed. The fundamental concepts of queue lengths and delay were introduced by Webster (1958) and Akçelik (1980). Their studies provided the cornerstones for the development of diverse methods for estimating queue lengths, including the conservation equation (Lindley, 1952; Miller, 1965), the shockwave theory (Lighthill and Whitham, 1955; Richards, 1956) and the probe vehicle approach (Comert and Cetin, 2009; Cheng et al., 2011, 2012; Christofa, 2013).

Methods based on the conservation equation have been widely adopted for the estimation of queue lengths over the past several decades due to their simplicity, robustness and applicability. For example, Chang and Su (1995) developed a neural network approach to examine the accuracy of queue-length estimates by using various levels of available detector data such as time intervals and numbers of lanes. Furthermore, Heidemann (1994) and Mung et al. (1996) used the distributions of queue lengths to improve the accuracy of estimates based on the conservation equation. Next, Mystkowski and Khan (1999) found that queue lengths tended to be overestimated in low traffic volume conditions. They compared the maximum queue lengths estimated by SIGNAL94, 
SYNCHRO3, TRANSYT-7F, PASSER II-90 and CORSIM. Likewise, Viloria et al. (2000) compared the estimations of queue lengths (as calculated by the accumulation and the discharge of vehicular queues) with the results from existing queue models (such as SIDRA, NETSIM, TRANSYT-7F, SOAP, SIGNAL 97 and HCM 2000). Wu et al. (2008) used detector occupancy data to revise queue lengths estimated at metered on-ramps, and compared the results with those derived from other queuelength estimation methods such as the Kalman filter, the HCM model or the random numbers approach. To overcome the accumulated errors induced by the conservation equation, Liu et al. (2007) developed an equation to estimate queue lengths by following a metering strategy that considered the levels of counting errors from both queue and passage detectors. Recently, Mucsi et al. (2011) used occupancy data to improve the accuracy of queue length estimates by adding mid-block detectors to an adaptive neuro-fuzzy inference system. Furthermore, Papamichail and Papageorgiou (2011) explained the interactions between queues generated at adjacent approaches on metered dual-branch on-ramps by balancing the relative queues with conservation equations and occupancy data.

In addition to the conservation equation, several other methods have been used for making real-time estimations of queue lengths at isolated signalized intersections. Probabilistic models have used different methods than deterministic models for estimating queue lengths. Viti and Van Zuylen (2004, 2010a, 2010b) performed analytic comparisons of traditional and probabilistic models. Vigos et al. (2008) and Vigos and Papageorgiou (2010) transformed time occupancy data into space occupancy data to reduce the accumulative counting errors and improve the accuracy of queue estimates. Furthermore, these authors applied the Kalman filter and additional mid-block detectors to estimate the queue lengths for each lane. Chung et al. (2012), Chang (2013) and Lee et al. (2013) introduced simple methods based on the conservation equation for using occupancy data that was collected from mid-block detectors to reduce the calculation time. To resolve the limitations of the conservation equation for oversaturated traffic conditions, Liu et al. (2009) calculated queue lengths by estimating the shockwave speed and by identifying break points based on the Lighthill-WhithamRichards (LWR) shockwave theory (Lighthill and Whitham, 1955; Richards, 1956). In recent years, Li et al. (2013) and Comert (2013) have used vehicle data to estimate vehicular queues, and they combined this method with the conventional estimation method. Lu and Chang (2012) and Lu and Yang (2014) estimated dynamic queue length distribution by integrating the classic queuing model and probability generating model.

Basically, the estimated lengths of queues have been used to measure delay, which was the most important measure of effectiveness (MOE) for ATCSs at isolated signalized junctions. Theoretically, measurements of delay were used to modify signal plans through changing factors such as the duration of green lights, cycle times and the structures of phases. Mirchandani and Head (2001) described a simple process for estimating queue lengths and making real-time predictions based on vehicle arrivals and queue discharge rates. The diverse real-time adaptive control algorithms introduced by Shelby (2004) have also been implemented to estimate real-time queue lengths by using the conservation equation. Li and Prevedouros (2004) estimated queue lengths as a means of developing traffic-adaptive control systems based on real-time turning proportions, arrival rates at upstream detectors and discharge rates at downstream detectors. Fang and Elefteriadou (2006) used the free-flow travel time between the advanced detectors ( $200 \mathrm{~m}$ from the stop-line) and the stop-line detectors to estimate the average queue lengths, and hence to calculate the minimum performance measure index (PMI) for the optimization of signal plans. Gartner et al. (2002) developed a different performance function for switching a stage of signal timing based on the conservation equation bound by the initial queue lengths, the arrivals and the discharge conditions. Moreover, a recent study by Zheng and Recker (2013) introduced an adaptive control algorithm that used combined arrival and 
discharge curves to estimate queue accumulation curves and to specify the real-time control objectives. In addition, one of the conservation equation-based approaches for measuring vehicle queues played an important role in the max pressure (MP) signal control policy developed by Varaiya (2013).

Recently, the group-based and lane-based optimization methods have provided more flexible specifications for signal timings in fixed-time control systems (Heydecker and Dudgeon, 1987; Wong, 1995, 1996; Wong and Wong, 2003; Wong et al., 2006). It appears promising to extend such methods of adaptive control logic for adjusting the durations, start times and sequences of signal groups for real-time control. Lee et al. (2014) made a first step toward estimating lane-to-lane turning flows at isolated signalized junctions on a real-time basis. The next step is to estimate the queue lengths on individual lanes at signalized junctions. Data on both the turning flows and queue lengths are essential inputs for the development of adaptive control logic. Therefore, this study aims to address the second step of devising a real-time estimation procedure for the queue lengths for individual lanes.

\section{METHODOLOGY}

This section discusses the formulation of the proposed method for using discriminant models, proportions of total traffic flows and downstream arrivals as estimated by a recursive process. The proposed data-processing method for the real-time estimation of lane-based queue lengths is illustrated in Figure 1. The detailed explanations for this proposed method are provided in the following sub-sections.

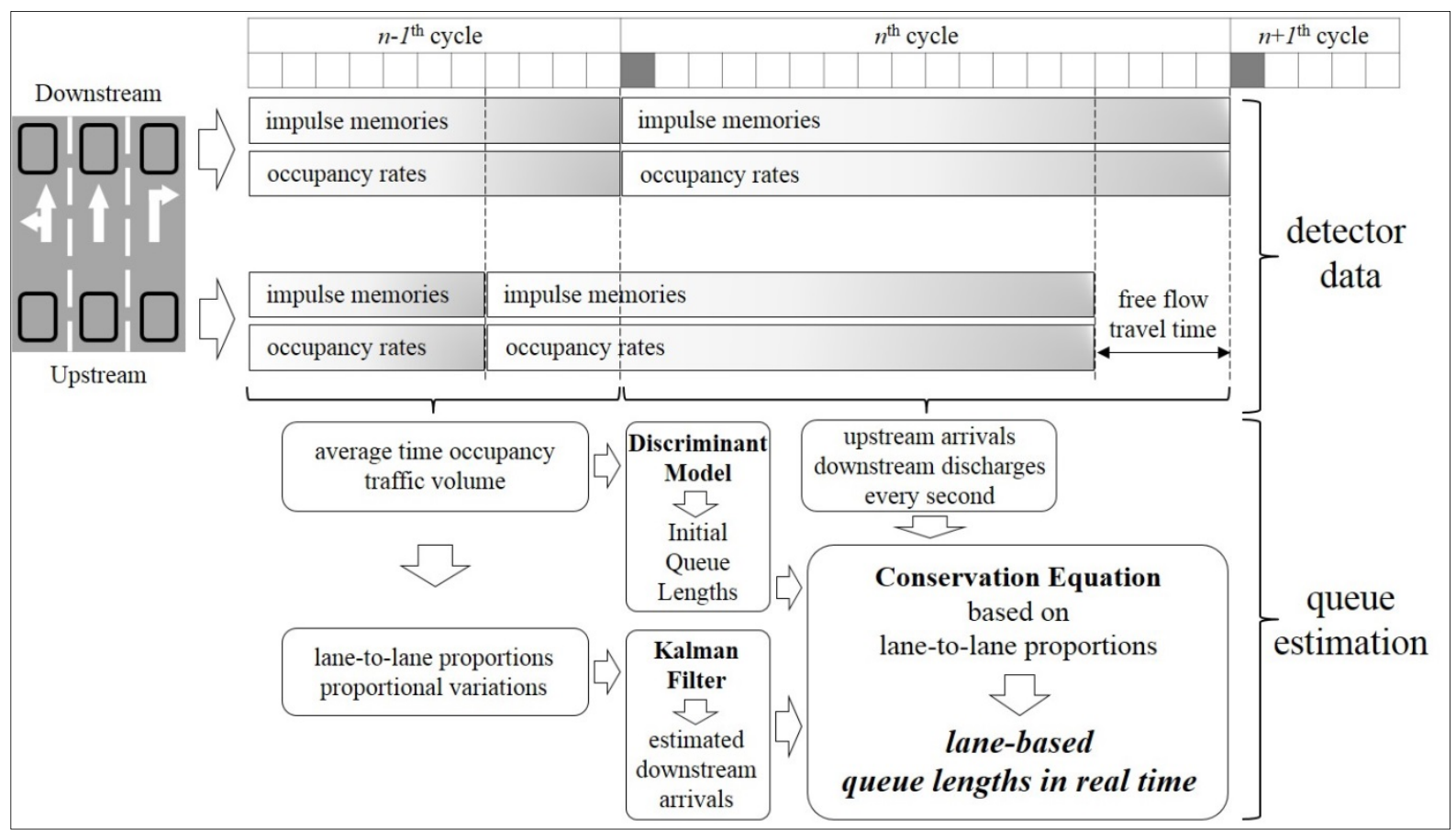

Figure 1. Data processing method for the real-time estimation of lane-based queue lengths

The definition of the indices, parameters and variables used in this study are given below.

$K \quad$ number of lanes

$N \quad$ number of cycles

$T \quad$ number of time steps in a cycle

$t_{\mathrm{f}} \quad$ the free-flow travel time from the upstream detector to the downstream detector

$o_{\mathrm{u}, k}^{n}\left(t-t_{\mathrm{f}}\right) \quad$ the time occupancy from the upstream detector in lane $k$ at time $t-t_{f}$ in the $n$th cycle

$o_{\mathrm{d}, k}^{n}(t) \quad$ the time occupancy from the downstream detector in lane $k$ at time $t$ in the $n$th cycle 
$i_{\mathrm{u}, k}^{n}\left(t-t_{\mathrm{f}}\right) \quad$ the upstream arrivals according to the binary impulse memory from the upstream detector in lane $k$ at time $t-t_{\mathrm{f}}$ in the $n$th cycle

$i_{\mathrm{d}, k}^{n}(t) \quad$ the downstream discharges according to the binary impulse memory from the downstream detector in lane $k$ at time $t$ in the $n$th cycle

$\hat{a}_{\mathrm{d}, k}^{n}(t) \quad$ the estimated downstream arrivals according to the estimated arrival rates from the downstream detector in lane $k$ at time $t$ in the $n$th cycle

\subsection{Discriminant Model}

\subsubsection{Model formulation}

To develop the real-time estimation of lane-based queue lengths, the first step is to establish the discriminant models by logistic regression. These models identify the existence of residual queues in each lane at the start time of every cycle and prevent the cumulative errors induced by passage detectors or counting errors. The probability of the existence of a residual queue in lane $k$ at start time $t$ in cycle $n$ is specified as

$$
\operatorname{Prob}\left\{x_{k}^{n}(t)=1\right\}=\frac{1}{1+\exp \left(-u_{k}^{n}(t)\right)},
$$

where $x_{k}^{n}(t)$ is a binary variable with a value of either " 1 " or " 0 " to identify the existence or absence of a residual queue, respectively. Moreover, $u_{k}^{n}(t)$ is the logit (or utility function) that is defined as

$$
\begin{aligned}
& u_{k}^{n}(t)=\alpha+\beta_{1} \frac{1}{m} \sum_{t=T-m+1}^{T} o_{\mathrm{d}, k}^{n-1}(t)+\beta_{2} \sum_{k=1}^{K} \sum_{t=1}^{R} i_{\mathrm{u}, k}^{n-1}\left(t-t_{\mathrm{f}}\right) \times p_{\mathrm{d}, k}^{n-1}+\beta_{3} \sum_{k=1}^{K} \sum_{t=T-R}^{T} i_{\mathrm{u}, k}^{n-1}\left(t-t_{\mathrm{f}}\right) \times p_{\mathrm{d}, k}^{n-1} \\
& +\beta_{4} \frac{1}{K} \frac{1}{T} \sum_{k=1}^{K} \sum_{t=1}^{T} o_{\mathrm{u}, k}^{n-1}\left(t-t_{\mathrm{f}}\right) \\
& \text { and } \quad p_{\mathrm{d}, k}^{n-1}=\frac{\sum_{t=1}^{T} i_{\mathrm{d}, k}^{n-1}(t)}{\sum_{k=1}^{K} \sum_{t=1}^{T} i_{\mathrm{d}, k}^{n-1}(t)}
\end{aligned}
$$

In Equation (2), $\beta_{1} \frac{1}{m} \sum_{t=T-m+1}^{T} o_{\mathrm{d}, k}^{n-1}(t)$ is the average time occupancy rate, which is collected by the downstream detector on lane $k$ during the effective time duration $m$ in the $(n-1)$ th cycle. The average time occupancy rate can explain how the discharging speeds and rates at a stop line influence the possibility of a residual queue. $\beta_{2} \sum_{k=1}^{K} \sum_{t=1}^{R} i_{\mathrm{u}, k}^{n-1}\left(t-t_{\mathrm{f}}\right) \times p_{\mathrm{d}, k}^{n-1}$ and $\beta_{3} \sum_{k=1}^{K} \sum_{t=T-R+1}^{T} i_{\mathrm{u}, k}^{n-1}\left(t-t_{\mathrm{f}}\right) \times p_{\mathrm{d}, k}^{n-1}$ are the traffic flows during the red and green periods, respectively, which can be calculated from upstream detector data in the $(n-1)$ th cycle. $R$ is the end time of the red signal for lane $k$, and $T-R+$ 1 is the start time of the green signal for lane $k$. The reason why we set the traffic flows for red and green periods separately is that we consider the different levels of effectiveness in traffic flows for the red and green periods in terms of utility. The proportion of downstream discharges in each lane, $p_{\mathrm{d}, k}^{n-1}$, 
is used to examine the lane-changing behavior. Then, the last term, $\beta_{4} \frac{1}{K} \frac{1}{T} \sum_{k=1}^{K} \sum_{t=1}^{T} o_{\mathrm{u}, k}^{n-1}\left(t-t_{\mathrm{f}}\right)$, is the average time occupancy rate, which is collected from the upstream detectors during the $(n-1)$ th cycle. To consider the lane-changing behavior between the upstream and the downstream detectors, aggregated time occupancy rates are applied across all of the lanes between the start time $t$ and the free flow travel time $t_{\mathrm{f}}$.

\subsubsection{Model calibration}

To calibrate the discriminant models, the time occupancy and impulse memories at the upstream and downstream points are collected at the target intersection. After calibration, the parameters $\alpha, \beta_{1}, \beta_{2}, \beta_{3}$ and $\beta_{4}$ in Equation (2) are obtained. The logit $u_{k}^{n}(t)$ represents the likelihood of a residual queue in lane $k$ at the start time of cycle $n$. If $\operatorname{Prob}\left\{x_{k}^{n}(t)=1\right\}>0.5$, then we set the initial queue in lane $k$ at the start time of the present cycle $n$ as equal to the queue in this lane at the end time of the previous cycle $n-1$; otherwise, the initial queue is set as zero.

\subsection{Conservation Equation Based on Lane-to-Lane Proportions}

The traditional conservation equation is the basic form for the real-time estimation of lanebased queue lengths. To deal with the possibility of counting and passage errors, the number of queued vehicles in lane $k$ at time $t$ in the $n$th cycle, $q_{k}^{n}(t)$ is the maximum value between the conservation equation and zero, as specified in the following equation.

$$
q_{k}^{n}(t)= \begin{cases}\max \left\{x_{k}^{n}(t) q_{k}^{n-1}(T)+i_{\mathrm{u}, k}^{n}\left(t-t_{\mathrm{f}}\right)-i_{\mathrm{d}, k}^{n}(t), 0\right\}, & \text { for } t=1 \\ \max \left\{q_{k}^{n}(t-1)+i_{\mathrm{u}, k}^{n}\left(t-t_{\mathrm{f}}\right)-i_{\mathrm{d}, k}^{n}(t), 0\right\}, & \text { for } t \neq 1\end{cases}
$$

In Equation (4), the first equation is used for the start time of each cycle, whereas the second equation is used for other time intervals. $q_{k}^{n-1}(T)$ is the number of queued vehicles in lane $k$ at the last time point $T$ in the $(n-1)$ th cycle, and $x_{k}^{n}(t)$ is the output of the discriminant models. For example, if there is a residual queue in lane $k$ at time $T$ in the $(n-1)$ th cycle, then $x_{k}^{n}(t)=1$, and otherwise 0 . Furthermore, let $J$ be the set of upstream lanes, and $K$ be the set of downstream lanes.

\subsubsection{Modification of the conservation equation}

To account for the lane-changing behavior and counting errors, the figure for estimated downstream arrivals, $\hat{a}_{\mathrm{d}, k}^{n}(t)$, is used to replace the figure for upstream arrivals in Equation (4). The modified conservation equation is therefore given as

$$
q_{k}^{n}(t)= \begin{cases}\max \left\{x_{k}^{n}(t) q_{k}^{n-1}(T)+\hat{a}_{\mathrm{d}, k}^{n}(t)-i_{\mathrm{d}, k}^{n}(t), 0\right\}, & \text { for } t=1 \\ \max \left\{q_{k}^{n}(t-1)+\hat{a}_{\mathrm{d}, k}^{n}(t)-i_{\mathrm{d}, k}^{n}(t), 0\right\}, & \text { for } t \neq 1\end{cases}
$$

The estimated downstream arrivals, $\hat{a}_{\mathrm{d}, k}^{n}(t)$, are calculated by four different methods for determining the proportion of traffic flows. 
The first method is based on the proportion of the total downstream discharges, $d_{\mathrm{d}, k}^{n}$, in the following equation.

$$
\hat{a}_{\mathrm{d}, k}^{n}(t)=\sum_{k=0}^{K} i_{\mathrm{u}, k}^{n}\left(t-t_{\mathrm{f}}\right) \times d_{\mathrm{d}, k}^{n-1},
$$

where $d_{\mathrm{d}, k}^{n}=\frac{\sum_{t=1}^{T} i_{\mathrm{d}, k}^{n}(t)}{\sum_{k=1}^{K} \sum_{t=1}^{T} i_{\mathrm{d}, k}^{n}(t)}$. Equation (6) compares the proportion of downstream discharges in lane $k$ (collected in the $(n-1)$ th cycle) to the proportion of upstream arrivals.

The second method is based on the proportion of lane-to-lane traffic volume in the following equation.

$$
\hat{a}_{\mathrm{d}, k}^{n}(t)=\sum_{k=1}^{K}\left\{i_{\mathrm{u}, k}^{n}\left(t-t_{\mathrm{f}}\right) \times \frac{v_{j k}^{n-1}}{\sum_{j=1}^{J} v_{j k}^{n-1}}\right\}
$$

In equation (7), $v_{j k}^{n-1}$ is the proportion of lane-to-lane traffic volume from the upstream detector $j$ to the downstream detector in lane $k$ at time $t$ in the $(n-1)$ th cycle, which is specified as

Condition (1): when $k=j$, and $a_{\mathrm{u}, k}^{n-1} \neq 0$, then

$$
v_{k k}^{n-1}=\left\{\begin{array}{l}
2-\frac{a_{\mathrm{u}, k}^{n-1}+\frac{1}{2}\left[\left\{a_{\mathrm{u}, k}^{n-1}-d_{\mathrm{d}, k}^{n-1}\right\}+\mid a_{\mathrm{u}, k}^{n-1}-d_{\mathrm{d}, k}^{n-1}\right]}{a_{\mathrm{u}, k}^{n-1}} . \\
0, \text { otherwise }
\end{array}\right.
$$

Condition (1) is for vehicles that do not change lane moving from the upstream to the downstream detectors, $k=j$. If a vehicle is detected on the upstream detector, the proportion of lane-to-lane traffic volume, $v_{j k}^{n-1}$, can be expressed as the equation, which is composed of the proportion of upstream arrivals, $a_{\mathrm{u}, k}^{n-1}$, and the downstream discharges, $d_{\mathrm{d}, k}^{n-1}$. To consider both upstream arrivals larger and smaller than downstream arrivals, $\frac{1}{2}\left[\left\{a_{\mathrm{u}, k}^{n-1}-d_{\mathrm{d}, k}^{n-1}\right\}+\left|a_{\mathrm{u}, k}^{n-1}-d_{\mathrm{d}, k}^{n-1}\right|\right]$ is calculated and added to the upstream arrivals. Then, to prevent over- or underestimation, the value, which is divided by upstream arrivals to calculate the proportions, is subtracted from 2 .

Condition (2): when $k \neq j$, and $a_{\mathrm{u}, k}^{n-1} \neq 0$, then 


$$
v_{j k}^{n-1}=\left\{\begin{array}{ll}
1-v_{k k}^{n-1} & , \text { for } a_{\mathrm{u}, j}^{n-1}-d_{\mathrm{d}, j}^{n-1}>0, a_{\mathrm{u}, k}^{n-1}-i_{\mathrm{d}, k}^{n-1}(t)<0, \text { and } a_{\mathrm{u}, m}^{n-1}-d_{\mathrm{d}, m}^{n-1}>0, m \text { is the rest lane } \\
\frac{d_{\mathrm{d}, k}^{n-1}-a_{\mathrm{u}, j}^{n-1}}{a_{\mathrm{u}, j}^{n-1}} & , \text { for } a_{\mathrm{u}, j}^{n-1}-d_{\mathrm{d}, j}^{n-1}>0, a_{\mathrm{u}, k}^{n-1}-i_{\mathrm{d}, k}^{n-1}(t)<0, \text { and } a_{\mathrm{u}, m}^{n-1}-d_{\mathrm{d}, m}^{n-1} \leq 0, m \text { is the rest lane } \\
0 & , \text { otherwise }
\end{array} .\right.
$$

Condition (2) is for vehicles that change lane moving from the upstream to the downstream detectors, $k=j$. If a vehicle is detected on the upstream detector, upstream arrivals are larger than downstream discharges on lane $j$, upstream arrivals are smaller than impulse memories on lane $k$ and upstream arrivals are larger than downstream discharges on rest lane $m$. Thus, we can simply calculate the proportion of lane-to-lane traffic volume, $v_{j k}^{n-1}$ by $v_{k k}^{n-1}$ subtracted from 1 . When upstream arrivals are smaller than and equal to downstream discharges on rest lane $m$, the difference between the downstream discharges on lane $k$ and the upstream arrivals on lane $j$ is divided by the upstream arrivals on lane $j$. The proportions of upstream arrivals in lanes $k$ and $j$ during the $n$th cycle are $a_{\mathrm{u}, k}^{n}=\frac{\sum_{t=1}^{T} i_{\mathrm{u}, k}^{n}\left(t-t_{\mathrm{f}}\right)}{\sum_{k=1}^{K} \sum_{t=1}^{T} i_{\mathrm{u}, k}^{n}\left(t-t_{\mathrm{f}}\right)}$ and $a_{\mathrm{u}, j}^{n}=\frac{\sum_{t=1}^{T} i_{\mathrm{u}, j}^{n}\left(t-t_{\mathrm{f}}\right)}{\sum_{j=1}^{J} \sum_{t=1}^{T} i_{\mathrm{u}, j}^{n}\left(t-t_{\mathrm{f}}\right)}$, respectively.

In Equations (8) and (9), the data on the proportions of upstream arrivals and downstream discharges (as collected in the $(n-1)$ th cycle) are used to obtain $\hat{a}_{\mathrm{d}, k}^{n}(t)$. After this, a recursive process using the Kalman filter (Kalman, 1960) is applied on the third and fourth methods, based on the first and second methods, respectively, to improve the accuracy of the estimated downstream arrivals. These third and fourth methods are discussed in the next section.

\subsection{Kalman Filter}

The Kalman filter is an effective and efficient recursive process for time series analysis which is used in signal processing. To start the recursive process, the system variables, time update equations and measurement equations are set. The proportion of downstream arrivals, $a_{\mathrm{d}, k}^{n}$, and the proportion of downstream discharges, $d_{\mathrm{d}, k}^{n-1}$, are defined as the state variable and the measurement variable for cycle $n$, respectively. The recursive process of the Kalman filter, as used by Bishop and Welch (2001) in estimating $\hat{a}_{\mathrm{d}, k}^{n}$, is described in Table 1. $\hat{a}_{\mathrm{d}, k}^{n}$ is then used to estimate $\hat{a}_{\mathrm{d}, k}^{n}(t)$ instead of $d_{\mathrm{d}, k}^{n-1}$ in Equation (6). The third method for estimating downstream arrivals is given in the following equation (10).

$$
\hat{a}_{\mathrm{d}, k}^{n}(t)=\sum_{k=0}^{K} i_{\mathrm{u}, k}^{n}\left(t-t_{\mathrm{f}}\right) \times \hat{a}_{\mathrm{d}, k}^{n} .
$$

Likewise, we can obtain $\hat{v}_{j k}^{n}$ by applying the Kalman filter on the proportion of lane-to-lane traffic volume instead of $v_{j k}^{n-1}$ in Equations (7), (8) and (9). $\hat{v}_{j k}^{n-1}$ and $\hat{v}_{j k}^{n}$ are used in Table 1 as the 
measurement and the state variables on the recursive process of the Kalman filter instead of $d_{\mathrm{d}, k}^{n-1}$ and $a_{\mathrm{d}, k}^{n}$, respectively. The fourth method is outlined in the following equation (11).

$$
\hat{a}_{\mathrm{d}, k}^{n}(t)=\sum_{k=1}^{K}\left\{i_{\mathrm{u}, k}^{n}\left(t-t_{\mathrm{f}}\right) \times \frac{\hat{v}_{j k}^{n}}{\sum_{k=1}^{J} \hat{v}_{j k}^{n}}\right\} .
$$

Table 1. The recursive process of the Kalman filter

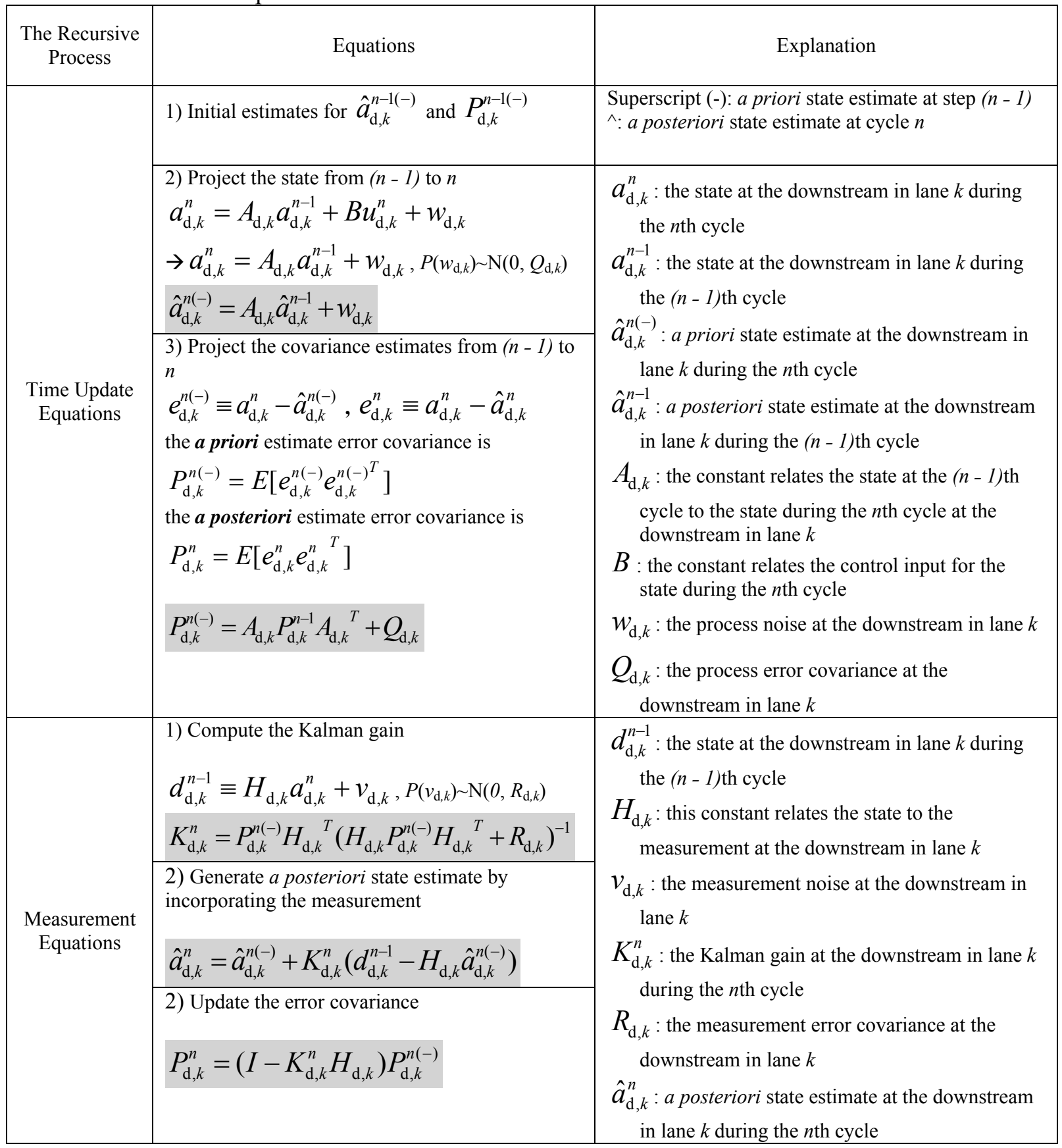




\section{4}

\section{Estimation Procedures}

To demonstrate the distinctive strength of the developed methods, a comparison of the various estimations of lane-based queue lengths based on various model structures is presented in the next section of this study. The specifications of the proposed models are described in Table 2.

Table 2. Descriptions of the proposed models based on the conservation equation

\begin{tabular}{|c|c|c|c|c|c|c|}
\hline Class & Model & $\begin{array}{c}\text { Conservation } \\
\text { equation }\end{array}$ & Reset & $\begin{array}{c}\text { Discriminant } \\
\text { model }\end{array}$ & Kalman filter & Proportions \\
\hline $\begin{array}{l}\text { Reference } \\
\text { model }\end{array}$ & $1^{*}$ & $\sqrt{ }$ & - & - & - & - \\
\hline \multirow{6}{*}{$\begin{array}{c}\text { Proposed } \\
\text { models }\end{array}$} & 2 & $\sqrt{ }$ & $\sqrt{ }$ & - & - & - \\
\hline & 3 & $\sqrt{ }$ & $\sqrt{ }$ & $\sqrt{ }$ & - & - \\
\hline & 4 & $\sqrt{ }$ & $\sqrt{ }$ & $\sqrt{ }$ & - & Equation (6) \\
\hline & 5 & $\sqrt{ }$ & $\sqrt{ }$ & $\sqrt{ }$ & - & Equation (7) \\
\hline & 6 & $\sqrt{ }$ & $\sqrt{ }$ & $\sqrt{ }$ & $\sqrt{ }$ & Equation (10) \\
\hline & 7 & $\sqrt{ }$ & $\sqrt{ }$ & $\sqrt{ }$ & $\sqrt{ }$ & Equation (11) \\
\hline
\end{tabular}

* The traditional conservation equation is used (Lindley, 1952; Miller, 1965; Mirchandani and Head, 2001; Fu et al. 2001; Liu et al., 2007)

Model 1 is the basic model, which is based on the traditional conservation equation for estimating the queue lengths, and which is commonly used to develop adaptive control logic models. Model 2 unconditionally resets the residual queue at the start time of every cycle, which prevents cumulative errors. Models 3 to 7 are the calibrated discriminant models, which have different functional forms for estimating the proportions of the downstream discharges and the upstream arrivals. For Model 3, the lane-changing behavior is not considered, and hence the upstream arrivals in each lane are used directly. Model 4, however, includes the proportions of downstream discharges in each lane, based on the data collected in the previous cycle. For Model 5, the proportions of the aggregated lane-to-lane traffic volumes in each lane are applied, as collected at the downstream detector in the previous cycle. For Models 6 and 7, the Kalman filter is applied to estimate the downstream arrivals and the lane-to-lane traffic volumes for the model formulation.

\section{MODEL RESULTS}

\subsection{Example Settings}

In this study, the performance of a typical directional dual three-lane section at an isolated intersection was simulated by using the VISSIM computer software. The vehicle-actuated programming in VISSIM was used to generate the impulse memories and time occupancy rates of the upstream and the downstream detectors. A four-phase signalized intersection with a cycle length of 60 seconds was created. The green signal duration for each phase was 10 seconds, with an inter-green time of 5 seconds. A full set of $2 \mathrm{~m} \times 2 \mathrm{~m}$ loop detectors was installed to record the impulse memory and time occupancy rate data. The upstream detectors were set back at distance $L$ from the stop-line. Left turns on red (LTOR) were prohibited in accordance with the driving rule for left-hand traffic. The geometric layout of the simulated intersection is presented in Figure 2. 


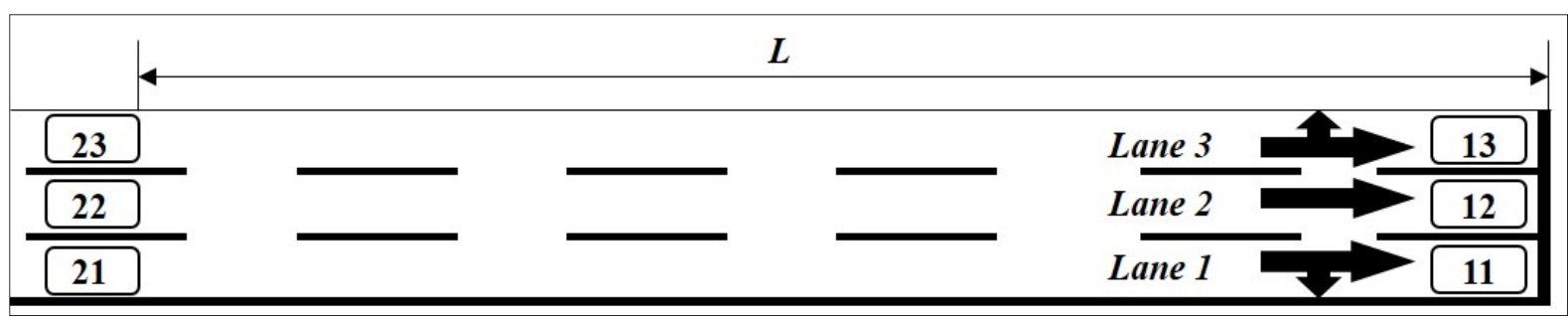

Figure 2. Geometric layout of the simulated intersection in VISSIM

The simulation period was 18,000 seconds and the simulation resolution was 1 second. Furthermore, the first and last 30 signal cycles were the warming up and warming down periods, respectively. Then, the saturation flow of this approach, which is calculated on the simulation, is 874 vehicles per hour (vph). Seven levels of traffic volume (degree of saturation), i.e., 700 (0.8), 750 (0.86), 800 (0.92), 850 (0.97), 900 (1.03), 950 (1.09) and 1,000 (1.14) vph, were set on all of the approaches in each 2,000-second interval for model calibration and validation. We further assumed that the turning proportions were evenly distributed among all of the movements. One-third of the vehicles were assigned to each turning movement for model calibration, but the proportions were randomly generated among all of the movements for model validation.

\subsection{Discriminant Models}

SPSS 20.0 was used to calibrate three logistic regression models, one for each setback of 100 $\mathrm{m}, 200 \mathrm{~m}$ and $300 \mathrm{~m}$ of the upstream detectors, as specified in Equation (2). The stepwise method was adopted to identify the significant variables. The calibration results are tabulated in Table 3.

Table 1. The calibration results of the discriminant models

\begin{tabular}{|c|c|c|c|c|}
\hline \multirow{4}{*}{ Distance } & Coefficients & \multicolumn{3}{|c|}{ Lanes } \\
\cline { 2 - 5 } & $\alpha$ & $-6.602^{*}$ & 2 & 3 \\
\cline { 2 - 5 }$(00 \mathrm{~m}$ & $\beta_{1}$ & $14.787^{*}$ & $-3.143^{*}$ & $-5.562^{*}$ \\
\cline { 2 - 5 } & $\beta_{2}$ & $0.507^{*}$ & $6.479^{*}$ & $9.553^{*}$ \\
\cline { 2 - 5 } & $\beta_{3}$ & - & - & - \\
\cline { 2 - 5 } & $\beta_{4}$ & $24.369^{*}$ & - & - \\
\hline \multirow{4}{*}{$200 \mathrm{~m}$} & $\alpha$ & $-6.238^{*}$ & $14.245^{*}$ & $94.969^{*}$ \\
\cline { 2 - 5 }$\left(t_{f}=12 \mathrm{~s}\right)$ & $\beta_{1}$ & $14.677^{*}$ & $-5.271^{*}$ & $-4.936^{*}$ \\
\cline { 2 - 5 } & $\beta_{2}$ & $0.540^{*}$ & $5.206^{*}$ & $-196^{*}$ \\
\cline { 2 - 5 } & $\beta_{3}$ & - & - & - \\
\hline \multirow{4}{*}{$300 \mathrm{~m}$} & $\beta_{4}$ & $19.270^{*}$ & - & $97.605^{*}$ \\
\cline { 2 - 5 }$\left(t_{f}=18 \mathrm{~s}\right)$ & $\beta_{1}$ & $-5.640^{*}$ & $-5.326^{*}$ & $-5.155^{*}$ \\
\cline { 2 - 5 } & $\beta_{2}$ & $15.322^{*}$ & $6.364^{*}$ & - \\
\cline { 2 - 5 } & $\beta_{3}$ & - & $-0.445^{*}$ & - \\
\cline { 2 - 5 } & $\beta_{4}$ & - & - & $107.512^{*}$ \\
\hline
\end{tabular}

* $0.1 \%$ significance level

** $1 \%$ significance level

$* * * 5 \%$ significance level

In Equation (2), the number of lanes was $K=3$, and the u was $m=4$ seconds for time occupancy rates at the downstream. The effective time duration is identical across all cycles and the value is determined by trial and error based on the calibration set. The calibration results 
demonstrated that the constants $\alpha$ were negative, and the coefficients $\beta_{k}$ were positive, except $\beta_{2}$ for the $300 \mathrm{~m}$ upstream detector for Lane 2 (at the $0.1 \%$ significance level). For $\beta_{1}$, a high average time occupancy rate at the downstream in each lane 4 seconds before the end of the previous cycle was likely to create a residual queue at the start time of the present cycle. For $\beta_{2}$, a high upstream arrival during the previous cycle was likely to create a residual queue at the start time of the present cycle in Lane 1 at the $100 \mathrm{~m}$ and $200 \mathrm{~m}$ upstream detectors only. For $\beta_{4}$, a high average time occupancy rate at the upstream on each lane was likely to create a residual queue at the start time of the present cycle. However, the coefficients $\beta_{3}$ were not selected by the stepwise method, as illustrated in the discriminant model.

\subsection{System Parameters for the Kalman Filter}

\subsubsection{The proportion of the total traffic flow}

For the recursive process based on the Kalman filter, the system parameters $A_{\mathrm{d}, k}, Q_{\mathrm{d}, k}, H_{\mathrm{d}, k}$ and $R_{\mathrm{d}, k}$ were calibrated according to the proportion of the total traffic flow in each specific lane, and the proportion of lane-to-lane traffic volume. It was assumed that these factors were linearly related to each other and were normally distributed. For the proportion of the total traffic flow in each specific lane, $A_{\mathrm{d}, k}$ was the constant that related the proportion of downstream arrivals into lane $k$ during the $(n-$ 1)th cycle to the proportion of arrivals during the $n$th cycle in the equation $a_{\mathrm{d}, k}^{n}=A_{\mathrm{d}, k} a_{\mathrm{d}, k}^{n-1}+w_{\mathrm{d}, k}$, $P\left(w_{\mathrm{d}, k}\right) \sim \mathrm{N}\left(0, Q_{\mathrm{d}, k}\right) . Q_{\mathrm{d}, k}$ was the process error covariance at the downstream in lane $k$. In the equation $d_{\mathrm{d}, k}^{n-1} \equiv H_{\mathrm{d}, k} a_{\mathrm{d}, k}^{n}+v_{\mathrm{d}, k}, P\left(v_{\mathrm{d}, k}\right) \sim \mathrm{N}\left(0, R_{\mathrm{d}, k}\right), H_{\mathrm{d}, k}$ was the constant that related the proportion of downstream arrivals into lane $k$ in the $n$th cycle to the proportion of downstream discharges from lane $k$ in the $(n-1)$ th cycle. $R_{\mathrm{d}, k}$ was the measurement error covariance at the downstream in lane $k$. The calibration results of the system parameters are presented in Table 4.

Table 2. The calibration results of the system parameters for the Kalman filter

\begin{tabular}{|c|c|c|c|c|}
\hline & \multicolumn{4}{|c|}{ System parameter } \\
\hline$k$ & $A_{\mathrm{d}, k}$ & $Q_{\mathrm{d}, k}$ & $H_{\mathrm{d}, k}$ & $R_{\mathrm{d}, k}$ \\
\hline 1 & 0.9362 & 0.0147 & 0.9238 & 0.0176 \\
\hline 2 & 0.8918 & 0.0156 & 0.8929 & 0.0151 \\
\hline 3 & 0.9528 & 0.0163 & 0.9540 & 0.0160 \\
\hline
\end{tabular}

Both constants, $A_{\mathrm{d}, k}$ and $H_{\mathrm{d}, k}$, were approximately equal to 1 , and both error covariances, $Q_{\mathrm{d}, k}$ and $R_{\mathrm{d}, k}$, were approximately equal to 0 . These parameters were then used to estimate the proportion of traffic volumes in lane $k$ during the present cycle through the recursive process given in Table 1.

\subsubsection{The proportion of the lane-to-lane traffic volume}

For the proportion of lane-to-lane traffic volume, time update equations and measurement equations were specified as $v_{j k}^{n}=A_{j k} v_{j k}^{n-1}+w_{j k}$ and $v_{j k}^{n-1} \equiv H_{j k} v_{j k}^{n}+v_{j k}$, respectively. Similarly, these time and volume factors were linearly related to each other and were normally distributed as $P\left(w_{j k}\right) \sim \mathrm{N}\left(0, Q_{j k}\right)$ and $P\left(v_{j k}\right) \sim \mathrm{N}\left(0, R_{j k}\right)$, respectively. $A_{j k}$ was the constant that related the proportion of traffic volume from the upstream detector $j$ to the downstream detector in lane $k$ during the $(n-1)$ th cycle, and to the same proportion during the $n$th cycle. In addition, $Q_{j k}$ was the process error covariance in the proportion of traffic volume from upstream detector $j$ to the downstream detector in lane $k$. Furthermore, $H_{j k}$ was the constant that related the proportion of downstream arrivals into lane 
$k$ in the $n$th cycle to the proportion of traffic volume from the upstream detector $j$ to the downstream detector in lane $k$ during the $n$th cycle, as compared to the same proportions during the $(n-1)$ th cycle. $R_{j k}$ was the measurement error covariance in the proportion of traffic volume from the upstream detector $j$ to the downstream detector in lane $k$. The estimated system parameters for the proportion of lane-to-lane traffic volume are specified in Table 5.

Table 3. Estimated Kalman system parameters for lane-to-lane traffic flows

\begin{tabular}{|c|c|c|c|c|c|c|c|c|c|c|c|c|c|}
\hline \multicolumn{2}{|c|}{ 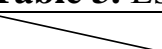 } & \multicolumn{4}{|c|}{$100 \mathrm{~m}$} & \multicolumn{4}{|c|}{$200 \mathrm{~m}$} & \multicolumn{4}{|c|}{$300 \mathrm{~m}$} \\
\hline$j$ & $k$ & $A$ & $Q$ & $H$ & $R$ & $A$ & $Q$ & $H$ & $R$ & $A$ & $Q$ & $H$ & $R$ \\
\hline \multirow{3}{*}{21} & 11 & 0.9805 & 0.0345 & 0.9805 & 0.0345 & 0.9450 & 0.0836 & 0.9450 & 0.0836 & 0.9432 & 0.0852 & 0.9432 & 0.0852 \\
\hline & 12 & 0.0568 & 0.0067 & 0.0568 & \begin{tabular}{|c|}
0.0067 \\
\end{tabular} & 0.1118 & 0.0059 & 0.1118 & 0.0059 & 0.1280 & 0.0055 & 0.1280 & 0.0055 \\
\hline & 13 & 1970 & 0.0111 & 0.1970 & 0.0111 & 0.3620 & 0.0435 & 0.3620 & 0.0435 & 0.3479 & 0.0429 & 0.3479 & 0.0429 \\
\hline \multirow{3}{*}{22} & 11 & 0.3447 & 0.0345 & 0.3447 & 0.0345 & 0.4090 & 0.0331 & 0.4090 & 0.0331 & 0.3049 & 0.0290 & 0.3049 & 0.0290 \\
\hline & 12 & 0.9224 & 0.1065 & 0.9224 & 0.1065 & 0.8912 & 0.1215 & 0.8912 & 0.1215 & 0.9000 & 0.1166 & 0.9000 & 0.1166 \\
\hline & 13 & 0.2698 & 0.0321 & 0.2698 & 0.0321 & 0.4474 & 0.0570 & 0.4474 & 0.0570 & 0.4353 & 0.0571 & 0.4353 & 0.0571 \\
\hline \multirow{3}{*}{23} & 11 & 0.1324 & 0.0074 & 0.1324 & 0.0074 & 0.0906 & 0.0053 & 0.0906 & 0.0053 & 0.0160 & 0.0048 & 0.0160 & 0.0048 \\
\hline & 12 & 0.1335 & 0.0075 & 0.1335 & 0.0075 & 0.0000 & 0.0006 & 0.0000 & 0.0006 & 0.0521 & 0.0004 & 0.0521 & 0.0004 \\
\hline & 13 & 0.9791 & 0.0358 & 0.9791 & 0.0358 & 0.9906 & 0.0180 & 0.9906 & 0.0180 & 0.9912 & 0.0169 & 0.9912 & 0.0169 \\
\hline
\end{tabular}

The highlighted cells in Table 5 represent the traffic flows without lane-changing. The constants were approximately equal to 1 , and the error covariances were approximately equal to 0 for these straight-moving traffic flows. However, the constants and the error covariances of other lane-tolane traffic flows were both approximately equal to 0 .

\subsection{Lane-to-Lane Estimation of Queue Lengths}

To measure the accuracy of the proposed model, the root mean square error (RMSE) was adopted in this study. The definition of the RMSE is given as,

$$
\varepsilon^{\mathrm{RMSE}}=\sqrt{\frac{\sum_{\forall k, t} \varepsilon_{k}(t)^{2}}{T \times K}},
$$

where $\varepsilon_{k}(t)=q_{k}^{\text {obs }}(t)-q_{k}^{\text {est }}(t)$ is the difference between the observed and the estimated lane-based queue lengths in lane $k$ at the time interval $t ; q_{k}^{\text {obs }}(t)$ is the observed queue length in lane $k$ at the time interval $t$, and $q_{k}^{\text {est }}(t)$ is the estimated queue length in lane $k$ at the time interval $t$, from Equations (4) and (5). $T$ is the number of time intervals $-14,400$ seconds (250 signal cycles) for the five levels of traffic volumes, excluding the warming up and warming down times. The queue capacity between the upstream detector and the downstream detector was 15,30 and 45 vehicles for the $100 \mathrm{~m}, 200 \mathrm{~m}$ and $300 \mathrm{~m}$ detectors, respectively. The RMSE values of the calibration results from the seven models are specified in Table 6.

Table 4. RMSE values of the calibration set

\begin{tabular}{|c|r|r|r|r|r|r|r|}
\hline $\begin{array}{c}\text { Distance from } \\
\text { stop line }\end{array}$ & Model 1 & Model 2 & Model 3 & Model 4 & Model 5 & Model 6 & Model 7 \\
\hline $100 \mathrm{~m}$ & 8.60 & 4.36 & 3.20 & 2.57 & 2.83 & $\mathbf{2 . 3 0}$ & 2.69 \\
\hline $200 \mathrm{~m}$ & 21.03 & 7.52 & 7.33 & 2.71 & 2.67 & 2.67 & $\mathbf{2 . 5 0}$ \\
\hline $300 \mathrm{~m}$ & 30.39 & 8.18 & 8.36 & 3.81 & 3.51 & 4.21 & $\mathbf{3 . 4 8}$ \\
\hline
\end{tabular}


The results demonstrated that Model 7 had the highest level of estimation accuracy. The RMSE values for this model were 2.69, 2.50 and 3.48 vehicles for the $100 \mathrm{~m}, 200 \mathrm{~m}$ and $300 \mathrm{~m}$ upstream detectors, respectively. In contrast, Model 1 had the lowest level of estimation accuracy, with RMSE values of 8.60, 21.03 and 30.39 vehicles for the $100 \mathrm{~m}, 200 \mathrm{~m}$ and $300 \mathrm{~m}$ upstream detectors, respectively. These results indicated that the proposed methods, based on the modified conservation equation and using the discriminant models and the Kalman filter, improved the accuracy of real-time estimation of lane-based queue lengths under diverse traffic situations.

\subsection{Validation}

To further validate the accuracy and the robustness of these models, five additional scenarios were randomly generated, with traffic volumes and turning proportions varying from $0 \%$ to $100 \%$ per turning flow. The total traffic volume was increased gradually from $700 \mathrm{vph}$ to $1,000 \mathrm{vph}$, to test the performance of the models under free-flow and congested conditions. The RMSE values of Models 1 to 7 for the five aggregated scenarios are described in Figure 3.

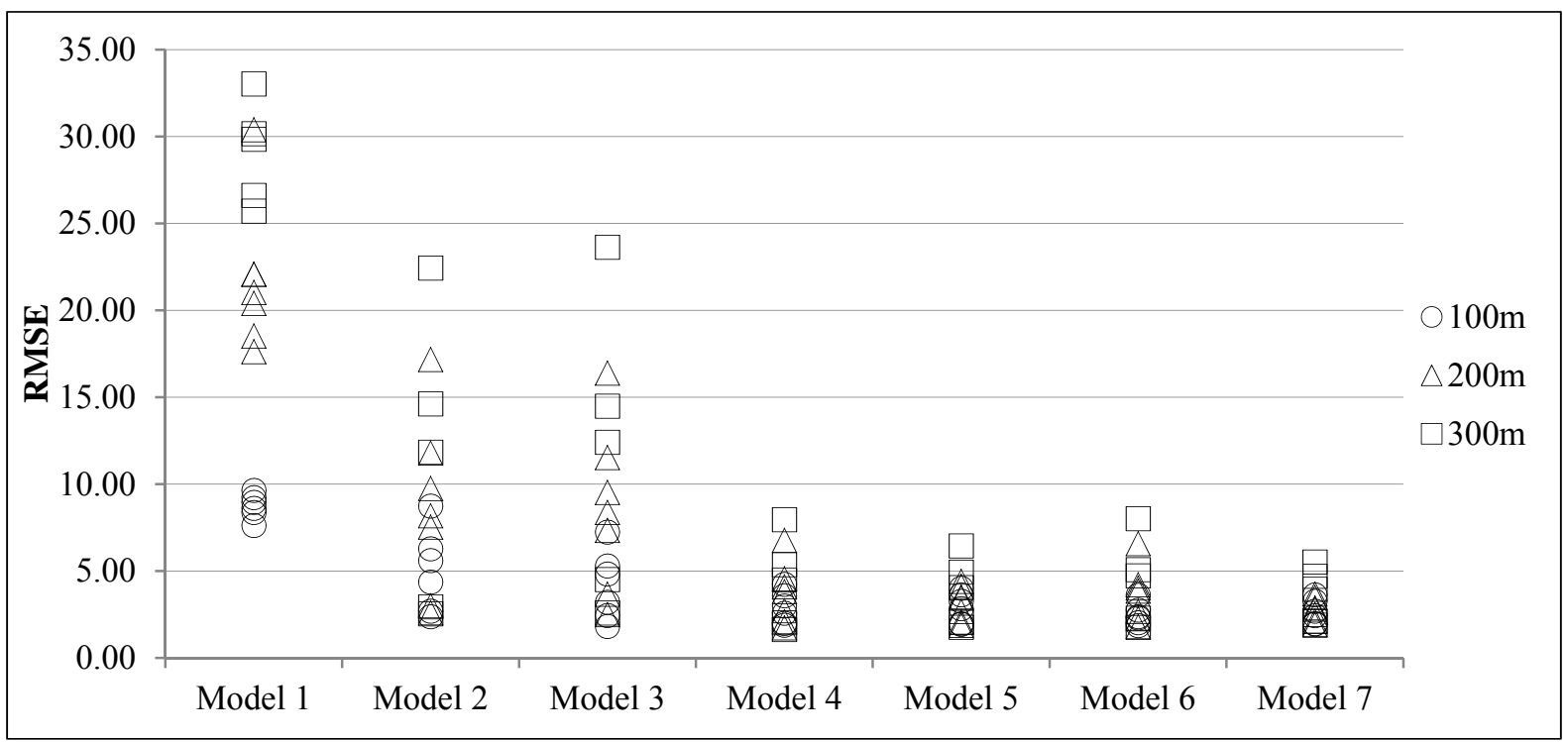

Figure 3. The RMSE values for the seven model formulations

In Figure 3, the RMSE values of Model 1 were distributed from 8 to 33 vehicles. The RMSE values of Models 2 and 3 were scattered between 2 and 25 vehicles, whereas those of Models 4 to 7 were scattered between 1 and 8 vehicles. For Models 1, 2 and 3, the RMSE values tended to mainly increase as the distance from the stop line to the upstream detectors increased. Among these models, Model 7 was the most stable, because the cumulative errors induced by lane-changing behavior and the counting errors were dramatically reduced by the discriminant model and the estimated downstream arrivals.

The average RMSE values for each model are specified in Table 7. The average RMSE values from Model 1 dramatically declined as the distance increased. The RMSE values from Models 2 and 3 doubled from the $100 \mathrm{~m}$ to the $300 \mathrm{~m}$ upstream detectors. Despite these changes in values, the accuracy of the models based on the discriminant model and the proportions of traffic volumes was more robust than the accuracy of the traditional conservation equation. Among the models developed in this study, Model 6 showed the highest accuracy for the estimation of lane-based queue lengths at the $100 \mathrm{~m}$ upstream detector, and Model 7 showed the greatest robustness and highest accuracy for queue length estimation at the $200 \mathrm{~m}$ and $300 \mathrm{~m}$ upstream detectors. 
Table 5. Average RMSE values of the validation sets

\begin{tabular}{|c|r|r|r|r|r|r|r|}
\hline $\begin{array}{c}\text { Distance from } \\
\text { stop line }\end{array}$ & Model 1 & Model 2 & Model 3 & Model 4 & Model 5 & Model 6 & Model 7 \\
\hline $100 \mathrm{~m}$ & 8.73 & 5.00 & 4.13 & 2.87 & 2.94 & $\mathbf{2 . 4 8}$ & 2.65 \\
\hline $200 \mathrm{~m}$ & 20.29 & 8.63 & 8.48 & 3.65 & 3.09 & 3.53 & $\mathbf{2 . 6 4}$ \\
\hline $300 \mathrm{~m}$ & 29.29 & 10.42 & 10.99 & 4.21 & 3.79 & 4.34 & $\mathbf{3 . 6 1}$ \\
\hline
\end{tabular}

The models that were based on the discriminant model and included lane-changing behavior (Models 4 to 7) showed noticeably improved accuracy. We therefore further analyzed the accuracy fluctuations of these models as traffic volumes increased at the stop line, and examined the tendency of RMSE values to aggregate over 10 cycles from 1,800 seconds to 16,200 seconds under different scenarios. A total of 3,600 RMSE values were calculated for each model, and the results are presented in Figure 4.

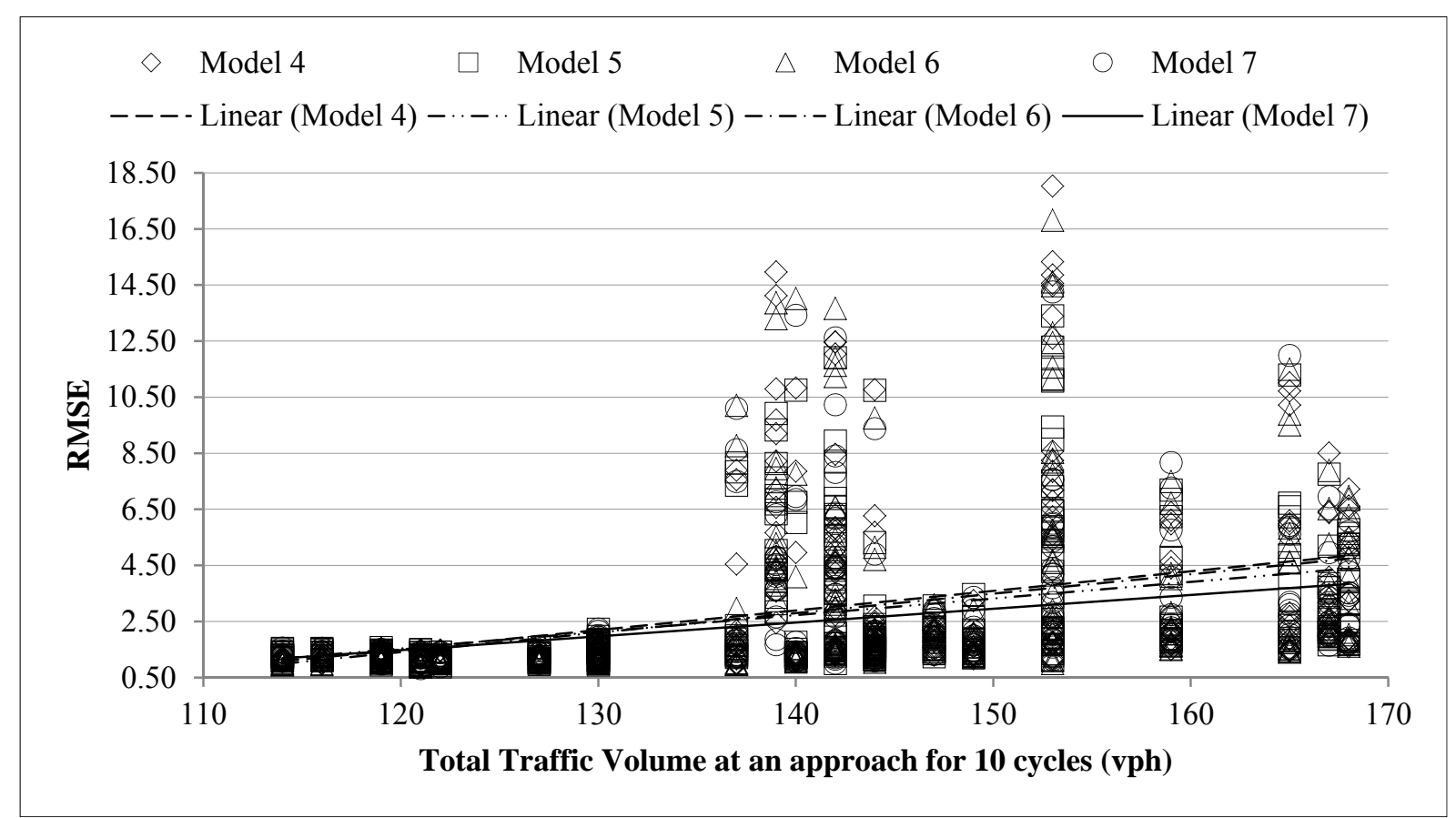

Figure 4. RMSE values for estimating lane-based queue lengths in the validation scenarios

In Figure 4, the RMSE values of all the models were scattered between 0.8 and 2.5 vehicles when the traffic volume at stop line was below 130 vph for 10 cycles ( 600 seconds). Furthermore, the RMSE values were widely distributed from 0.8 to 18.2 vehicles in the range of 135 to $155 \mathrm{vph}$, whereas the distributions narrowed down toward saturated conditions when the traffic volume was over $155 \mathrm{vph}$. In other words, all of the developed models showed relatively similar estimations of lane-based queue lengths at the lower traffic volumes, and the accuracy of the models tended to decrease as the traffic volume rose. Nonetheless, from the projection of the trend line, Model 7 still had the highest accuracy across all traffic conditions. In addition, the increase of RMSE values in Model 7 was lower than that for the other models as traffic volumes increased.

As illustrated in Figure 5, the observed and the estimated maximum queue lengths from Models 1 and 7 were subsequently compared, to demonstrate the effectiveness of the proposed method over that of the traditional conservation equation. For each lane, Model 7 estimated a maximum number of queued vehicles that was nearly identical to the observed number of queued 
vehicles. In Model 1, however, lane changing behavior was not explained, and it was found that the cumulative errors increased dramatically during a long-term analysis. In Model 7, the estimated maximum queue lengths were almost identical to the observed maximum queue lengths on the approach. Model 1, however, tended to overestimate the queue lengths.

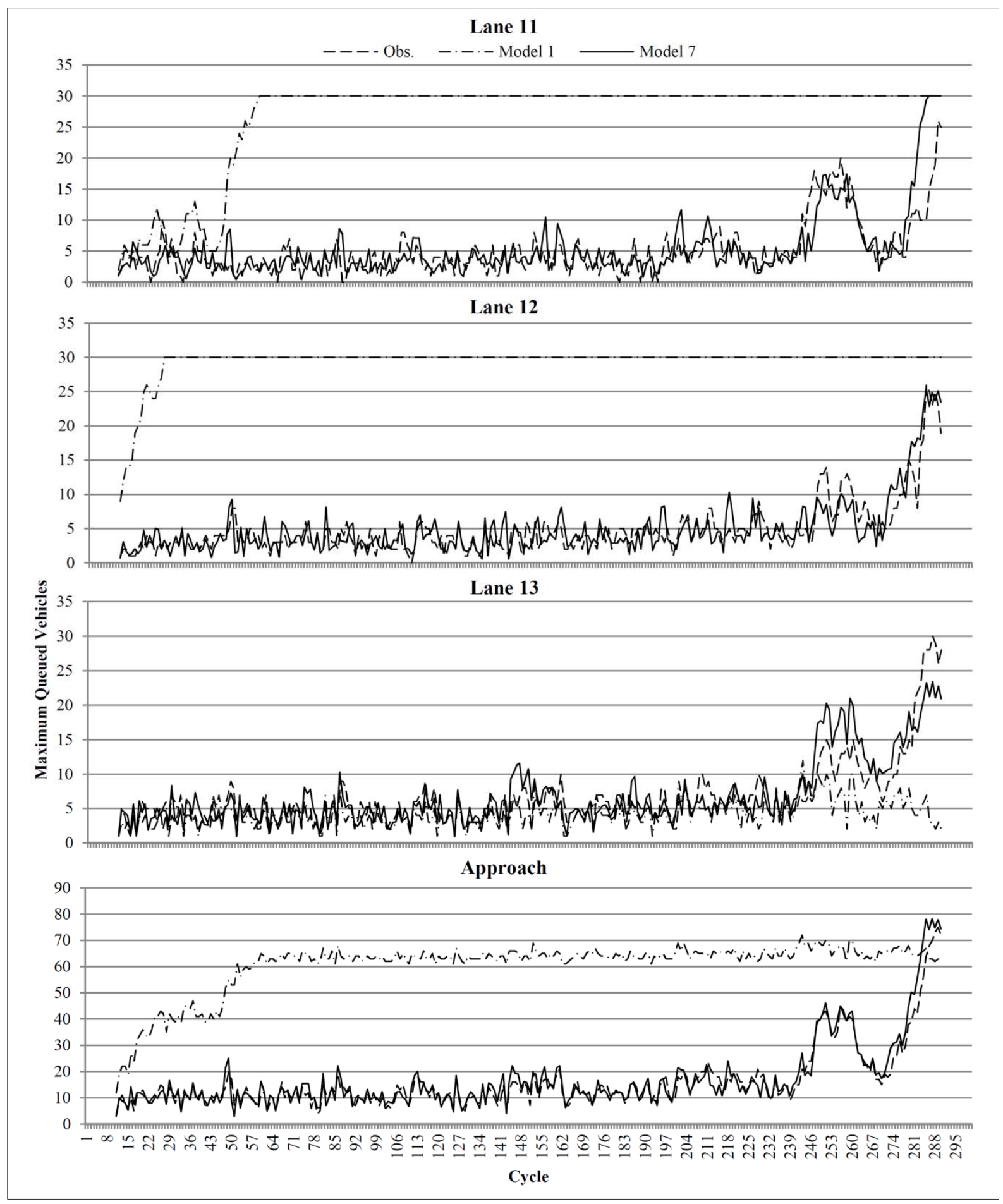

Figure 5. Maximum queue lengths as observed and as estimated by Models 1 and 7 in a validation set 


\section{CASE STUDY}

\subsection{Real Dataset}

In the case study, the developed estimation approaches were applied to a real junction at Lankershim Boulevard in Los Angeles, USA. The dataset and detailed information were provided by the Federal Highway Administration's Next Generation Simulation (NGSIM, 2006). The dataset was collected on June 16, 2005 from 8:28 a.m. to 9:00 a.m. by using five video cameras to establish the vehicle trajectory data. The junction of Lankershim Boulevard/ Campo De Cahuenga Way/ Universal Hollywood Drive (No. 2 intersection) was the target intersection for this study. An aerial photograph and the layout configuration of the target junction are shown in Figures 6(a) and 6(b), respectively. The recorded period had a total of 20 cycles, with a cycle length of 100 seconds. The data collected during the first 10 cycles were used to calibrate the discriminant models and the Kalman system parameters. The remaining data were used to validate the calibrated models.

The impulse memories and occupancy rates of the downstream and upstream detectors were computed based on the provided vehicle trajectory data (Vehicle ID, Frame ID, Local X and Y, Vehicle length, Velocity, Acceleration, Lane and Direction). Assuming that the vehicles moved linearly along the section, $2 \mathrm{~m} \times 2 \mathrm{~m}$ virtual detectors were installed by setting virtual lines offset $2 \mathrm{~m}$ from the start (downstream detectors) to the end (upstream detectors) of the section, respectively. The distance between the downstream and the upstream detectors was $167 \mathrm{~m}$. For impulse memories, Frame IDs and Lane data for each vehicle for each 1/10 of a second were extracted from the downstream and upstream detectors. For occupancy data from the upstream detectors, the durations from the Frame IDs of each vehicle between the section start and the virtual line were calculated, with consideration for Vehicle length, Velocity and Lane. Similarly, the occupancy data from the downstream detectors were the calculated durations from the Frame IDs of each vehicle between the section end and the virtual line.

To observe the real-time lane-based queue lengths, we computed the number of vehicles traveling below $5 \mathrm{~km} / \mathrm{h}$ as Velocity, and revised the length by checking the recorded video. The resulting impulse memories and occupancy rates from the downstream and upstream detectors were used for calibration and validation.

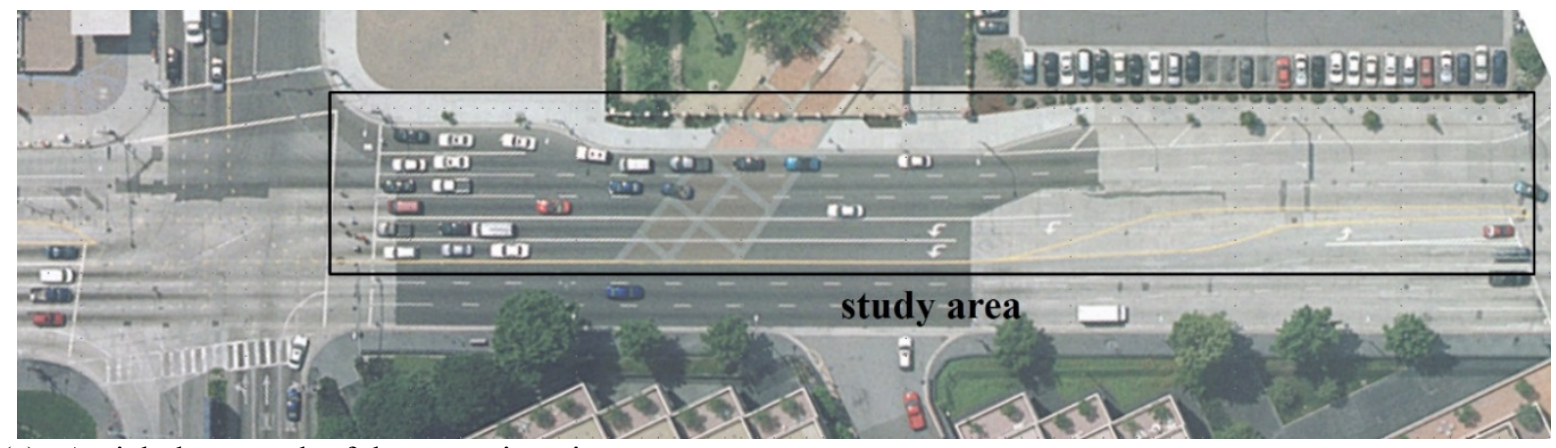

(a) Aerial photograph of the target junction 


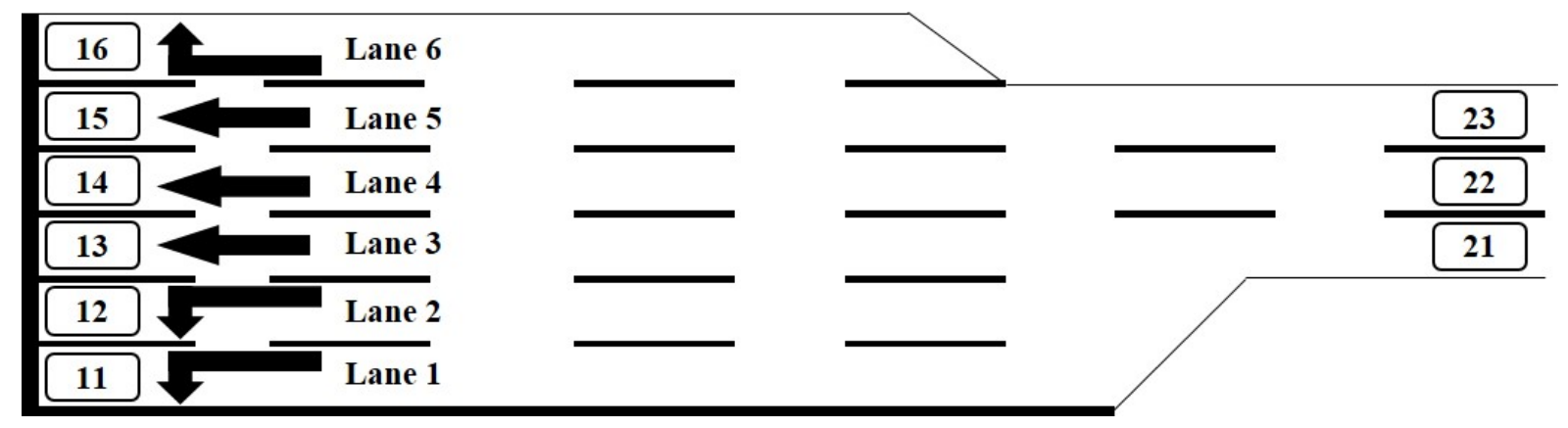

(b) Configuration of the target junction

Figure 6. Geometric conditions of the target intersection

\subsection{The Kalman System Parameters}

The Kalman system parameters were calibrated based on the calibration set. The proportions of the total traffic flow in each lane and the proportions of lane-to-lane traffic flow in each lane were used to calibrate the parameters. The calibration results are specified in Tables 8 and 9 .

Table 6. Estimated Kalman system variables for total traffic flows in the case study

\begin{tabular}{|c|c|c|c|c|}
\hline & \multicolumn{4}{|c|}{$167 \mathrm{~m}$} \\
\hline$k$ & $A$ & $Q$ & $H$ & $R$ \\
\hline 1 & 0.8002 & 0.0057 & 0.8002 & 0.0057 \\
\hline 2 & 0.9180 & 0.0056 & 0.9180 & 0.0056 \\
\hline 3 & 0.9139 & 0.0083 & 0.9139 & 0.0083 \\
\hline 4 & 0.9045 & 0.0093 & 0.9045 & 0.0093 \\
\hline 5 & 0.8436 & 0.0218 & 0.8436 & 0.0218 \\
\hline 6 & 0.7962 & 0.0016 & 0.7962 & 0.0016 \\
\hline
\end{tabular}

Table 7. Estimated Kalman system variables for lane-to-lane traffic flows in the case study

\begin{tabular}{|c|c|c|c|c|c|}
\hline & - & & 167 & & \\
\hline$j$ & $k$ & $A$ & $Q$ & $H$ & $R$ \\
\hline \multirow{6}{*}{21} & 11 & 0.7251 & 0.0186 & 0.7251 & 0.0186 \\
\hline & 12 & 0.8193 & 0.0288 & 0.8193 & 0.0288 \\
\hline & 13 & 0.9007 & 0.0468 & 0.9007 & 0.0468 \\
\hline & 14 & 0.0000 & 0.0007 & 0.0000 & 0.0007 \\
\hline & 15 & 0.0000 & 0.0001 & 0.0000 & 0.0001 \\
\hline & 16 & 0.7805 & 0.0031 & 0.7805 & 0.0031 \\
\hline \multirow{6}{*}{22} & 11 & 0.3895 & 0.0054 & 0.3895 & 0.0054 \\
\hline & 12 & 0.5498 & 0.0076 & 0.5498 & 0.0076 \\
\hline & 13 & 1.0000 & 0.0001 & 1.0000 & 0.0001 \\
\hline & 14 & 0.8855 & 0.1638 & 0.8855 & 0.1638 \\
\hline & 15 & 0.0000 & 0.0000 & 0.0000 & 0.0000 \\
\hline & 16 & 0.3668 & 0.0019 & 0.3668 & 0.0019 \\
\hline \multirow{6}{*}{23} & 11 & 0.7242 & 0.0023 & 0.7242 & 0.0023 \\
\hline & 12 & 0.7358 & 0.0063 & 0.7358 & 0.0063 \\
\hline & 13 & 1.0000 & 0.0001 & 1.0000 & 0.0001 \\
\hline & 14 & 0.0000 & 0.0001 & 0.0000 & 0.0001 \\
\hline & 15 & 0.9167 & 0.1062 & 0.9167 & 0.1062 \\
\hline & 16 & 0.7108 & 0.0012 & 0.7108 & 0.0012 \\
\hline
\end{tabular}


The highlighted cells in Table 9 represent traffic flows without lane-changing. Even if the calibration set of the real field data was sufficient to calibrate the Kalman system parameters, the data were not adequate to calibrate the discriminant models. Hence, to calibrate the discriminant models, VISSIM software was used to simulate the calibration set with sufficient numbers of average occupancy rates and traffic volumes for each cycle.

\subsection{Simulation for the Discriminant Models}

The signal plan, the turning proportions and the standard traffic flows data were established based on the real field data collected from 8:32:05 to 8:47:04 a.m., with 9 signal cycles to simulate the performance of the target intersection. The running time of the simulation was 14,400 seconds. A total of 124 signal cycles were used to calibrate the discriminant models, excluding the first and the last 10 cycles for warming up and down. The traffic volumes were in the range of $50 \%$ to $150 \%$ of the real traffic volumes, to provide a sufficient number of cycles, including the residual queues. The simulation layout is portrayed in Figure 7.

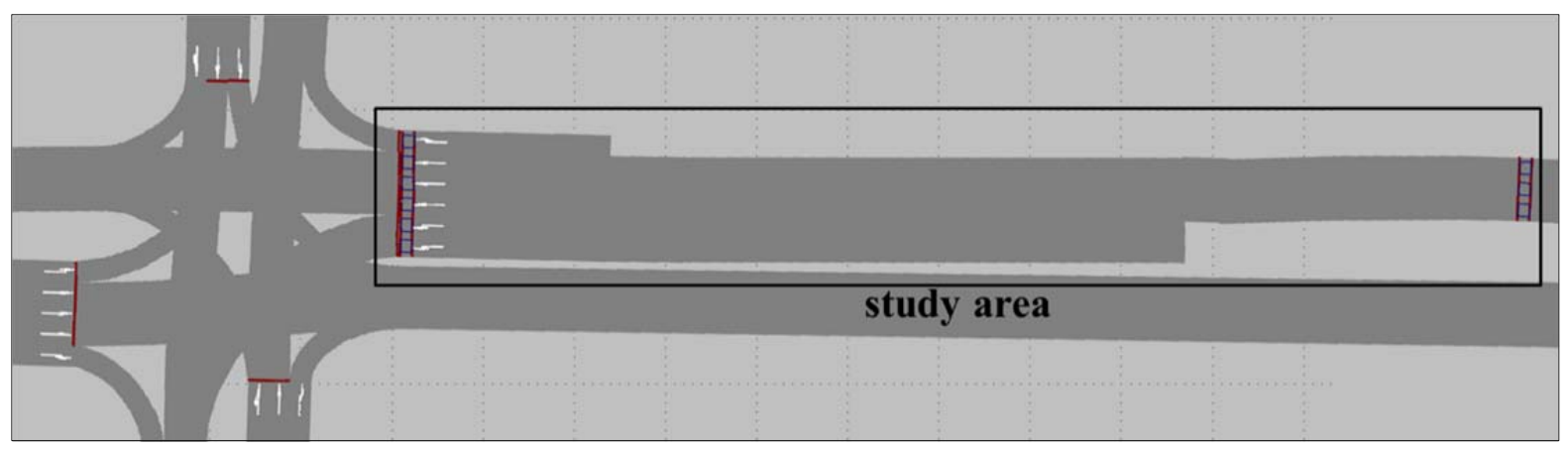

Figure 7. Simulation layout

The discriminant models for each lane were established based on the simulation data, and the results are specified in Table 10. To validate the effectiveness of the discriminant models based on VISSIM simulation, the real data were applied to the calibrated discriminant models for 18 cycles on both the calibration and validation sets. The discriminant models correctly identified the existence or absence of a residual queue in 96 out of 108 cases (6 lanes $\times 18$ cycles).

Table 8. The calibration results from the discriminant models for the case study

\begin{tabular}{|c|c|c|c|c|c|c|}
\hline \multirow{2}{*}{ Coefficients } & \multicolumn{6}{|c|}{ Lanes } \\
\hline & 1 & 2 & 3 & 4 & 5 & 6 \\
\hline$\alpha$ & $-5.334^{*}$ & $-5.376^{*}$ & $-7.537 *$ & $-6.255^{*}$ & $-9.755^{*}$ & $-2.108^{*}$ \\
\hline$\beta_{1}$ & $8.336^{*}$ & $10.751^{*}$ & - & - & - & - \\
\hline$\beta_{2}$ & $0.673^{*}$ & $0.625^{*}$ & - & - & $0.467 *$ & $0.803^{*}$ \\
\hline$\beta_{3}$ & - & - & - & $-0.253^{*}$ & - & $-0.496^{*}$ \\
\hline$\beta_{4}$ & - & - & $45.225^{*}$ & $63.012 *$ & $32.371^{*}$ & - \\
\hline $0.1 \%$ sign & ce level & & & & & \\
\hline $\begin{array}{ll}* * & 1 \% \text { signif } \\
* * * & 5 \% \text { signif }\end{array}$ & $\begin{array}{l}\text { level } \\
\text { level }\end{array}$ & & & & & \\
\hline
\end{tabular}

\subsection{The Results of the Case Study}

All of the estimated coefficients were at a $1 \%$ significance level, and there were reliable differences detected between the exclusive lanes for turning left, going, straight ahead and turning right. For the left-turning exclusive lanes (Lanes 1 and 2), the average occupancy rates during 4 seconds at the downstream detectors $\beta_{1}$ and the total traffic volumes during red times at the upstream 
detectors $\beta_{2}$ were significant. However, the average occupancy rates during the previous cycle for all of the upstream lanes $\beta_{4}$ were significant for the straight-moving traffic (Lanes 3, 4 and 5). Moreover, for the right-turning lane (Lane 6), the total traffic volumes during red and green times at the upstream detectors $\beta_{2}$ and $\beta_{3}$ were both significant.

The above-described calibrated models were then used to estimate the lane-based queue lengths compared to the calibration and the validation sets of the real field data. The average RMSE values of each model for both sets are specified in Table 11.

Table 9. The average RMSE values on the calibration and the validation sets

\begin{tabular}{|c|r|r|r|r|r|r|r|}
\hline & \multicolumn{1}{|c|}{ Model 1 } & \multicolumn{1}{c|}{ Model 2 } & \multicolumn{1}{c|}{ Model 3 } & \multicolumn{1}{c|}{ Model 4 } & Model 5 & \multicolumn{1}{c|}{ Model 6 } & Model 7 \\
\hline Calibration set & 85.429 & 10.554 & 10.554 & 5.669 & 5.007 & 5.442 & $\mathbf{4 . 7 9 4}$ \\
\hline Validation set & 83.966 & 15.019 & 15.019 & $\mathbf{5 . 6 8 3}$ & 7.459 & 6.235 & 6.427 \\
\hline
\end{tabular}

The results demonstrated that the accuracy of Model 7 was the highest on the calibration set (average RMSE value $=4.794$ ), whereas Model 4 had the highest accuracy to estimate lane-based queue lengths on the validation set (5.683). The accuracy of these models was dramatically improved after applying the discriminant model and the concept of proportions, whereas the accuracy of the models remained nearly the same in applying either the proportion of total traffic flows or the lane-tolane flows. Moreover, the Kalman filter increased the accuracy for the calibration set from Model 4 (5.669) to Model 6 (5.442) and from Model 5 (5.007) to Model 7 (4.794). However, the Kalman filter imposed a negative effect on the accuracy for the validation set from Model 4 (5.683) to Model 6 (6.235), and imposed a positive effect on the accuracy for the proportion of lane-to-lane traffic volume from Model 5 (7.459) to Model 7 (6.427). The RMSE values of each signal cycle in the calibration and validation sets for Models 4 to 7 were compared. The results are described in Figures 8 (a) and 8 (b).

The tendencies of the RMSE values for the developed models were almost the same according to cycles. For the calibration set, the accuracy of Models 5 and 7 (which included the discriminant model and the proportion of lane-to-lane traffic flows) were generally better than those of Models 4 and 6. The RMSE values for all of the models were scattered from 3 to 8 vehicles, whereas those of Model 7 were distributed from 3 to 5 vehicles across all cycles of the calibration set. The accuracy of models in the validation set was less than the accuracy of those in the calibration set, for which the values were scattered from 4 to 10 vehicles. Furthermore, the accuracy of Models 4 and 6 (which included the discriminant model and the proportion of total traffic flows) was slightly better than the accuracy of Models 5 and 7. The RMSE values of Model 4 were distributed from 4 to 8 vehicles, and this model had the highest accuracy across all cycles on the validation set. 


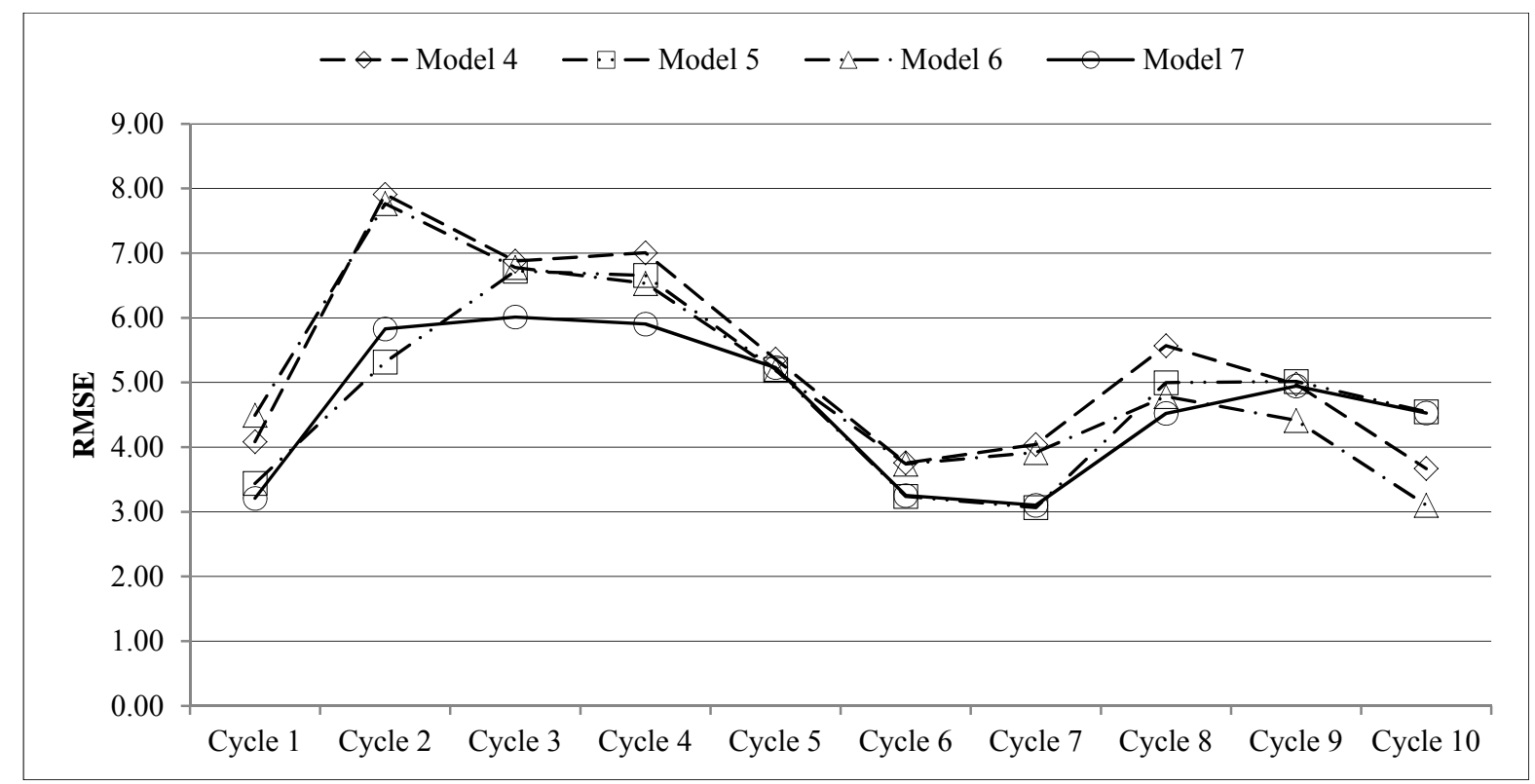

(a) RMSE values of each cycle in the calibration set

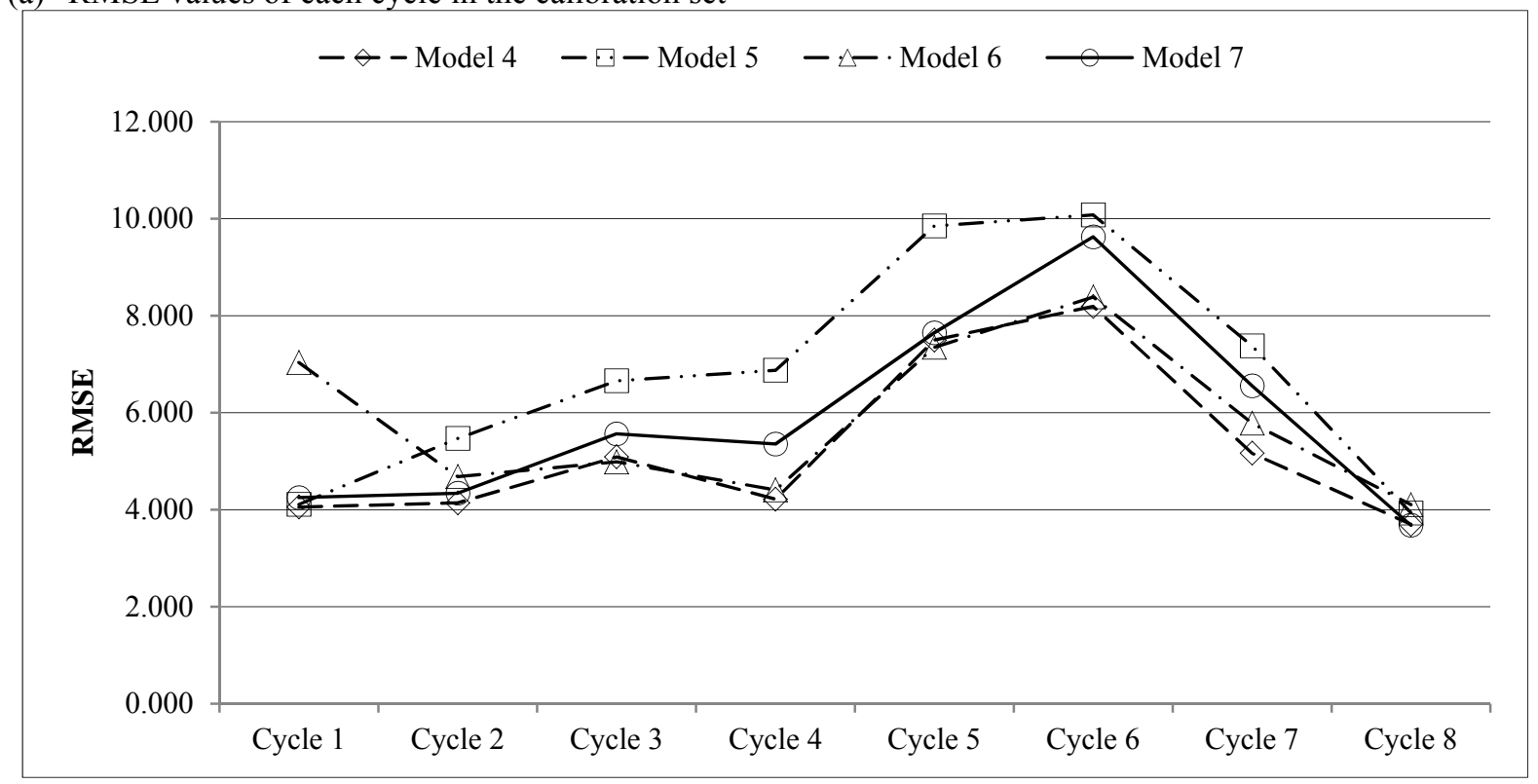

(b) RMSE values of each cycle in the validation set

Figure 8. The tendencies of RMSE values for the developed models

To examine the performance of the most effective model for a validation set of the case study, a comparison was made between the observed queue lengths and the queue lengths as estimated by Models 4 and 7. These values are described in Figure 9, in which Model 1 (based on the traditional conservation equation) is not presented, because the values of RMSE for this model were far larger than for the other models. For Lanes 11 and 12, which were the exclusive lanes for left-turning traffic, the estimated queue lengths tended to be slightly underestimated compared to the observed queue lengths. However, for the straight-ahead traffic (Lanes 13, 14 and 15), the estimation of lane-based queue lengths tended to be overestimated compared to the observed queue lengths. In addition, the queue lengths in Lane 16 did not have a distinctive pattern, because the total number of queued vehicles in this lane was small. The queue lengths on the approach tended to be slightly underestimated compared to the observed values, but the overall the estimations were almost identical to the observed values on the approach. 


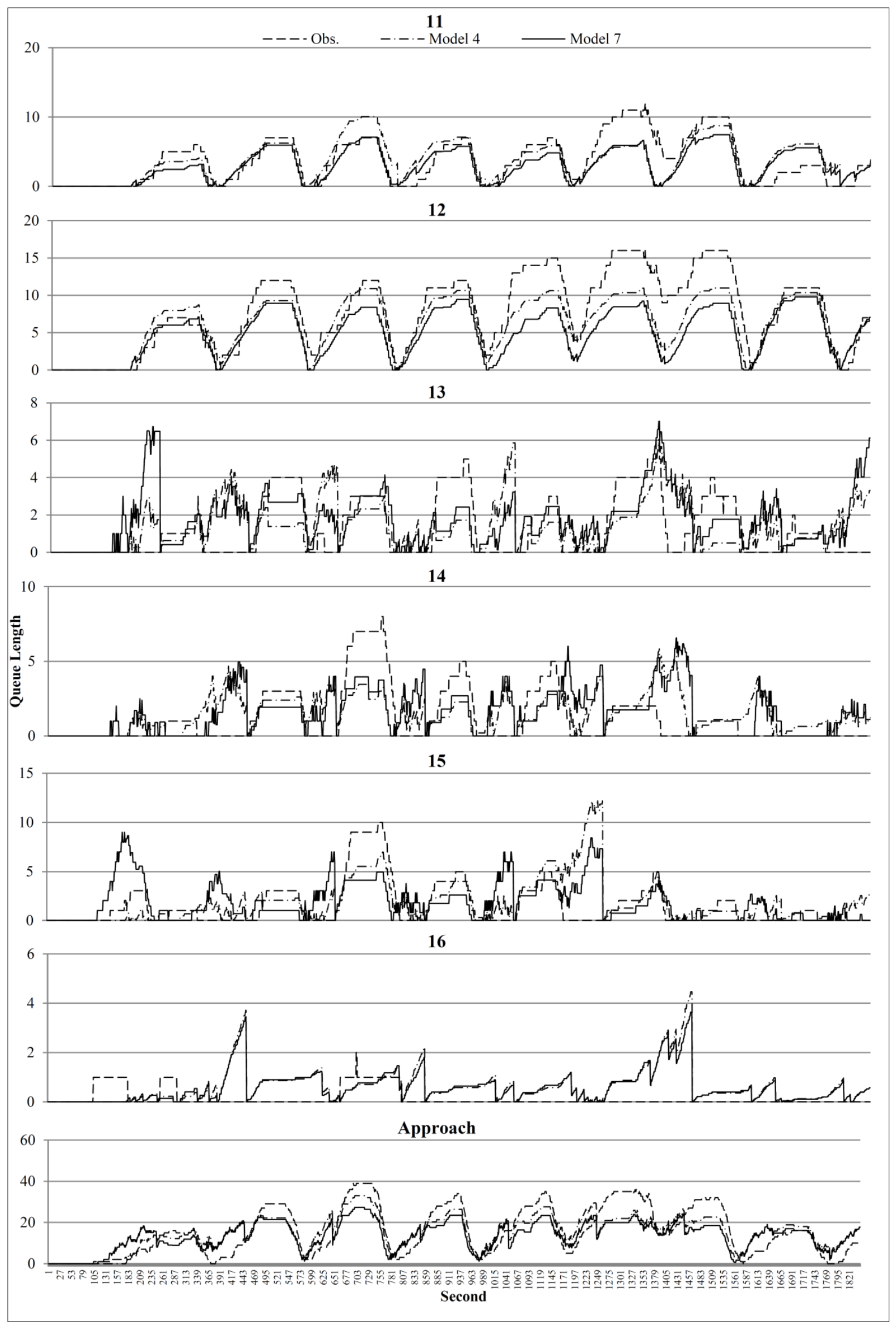

Figure 9. Comparison of queue lengths as observed and as estimated by Models 4 and 7 in the validation set of the case study 
According to the RMSE values and the tendency of estimated queue lengths in real time, the proposed models outperformed the traditional conservation equation for the estimation of lane-based queues. Even if the discriminant models as calibrated by the VISSIM simulation accurately estimated the residual queues at the start times of each phase for both the calibration and the validation sets on the real situations, the Kalman filter did not show any distinctive improvement on the effectiveness of the proposed models for the validation set.

\section{CONCLUSION}

In this study, a method for the real-time estimation of lane-based queue lengths was proposed. The key challenges were to identify whether there was a residual queue at the start time of each cycle, and to estimate the downstream arrivals for each lane. Discriminant models were estimated using time occupancy rates and impulse memories, based on the detector and the signal information from a set of upstream and downstream detectors. To determine the proportions of total traffic volume in each lane, the downstream arrivals in each lane for the present cycle were estimated using the Kalman filter, based on the upstream arrivals and the downstream discharges collected during the previous cycle.

In the simulations, seven models were examined, including the traditional conservation equation and the developed models that considered the discriminant models and the downstream arrivals as estimated by the Kalman filter. It was found that the calibrated discriminant models and the Kalman system parameters were effective. The proposed method performed well for the calibration set and for five different validation sets with $100 \mathrm{~m}, 200 \mathrm{~m}$ and $300 \mathrm{~m}$ upstream detectors. The results of the computer simulations showed that the real-time estimations of lane-based queue lengths based on the discriminant models and downstream arrivals as estimated by the Kalman filter outperformed the other methods. Moreover, the distance between the stop lines and the upstream detectors was an important factor for estimating the lane-based queue length, and $200 \mathrm{~m}$ upstream detectors were appropriate for the proposed method. This approach provided a sufficient capacity for queued vehicles and maintained a high accuracy of estimates. In a case study, the proposed models proved to be more accurate than the traditional models. Therefore, Model 7 (which considered the discriminant models, the Kalman filter and the proportions of lane-to-lane traffic flows) was recommended with $200 \mathrm{~m}$ upstream detectors. In addition, Model 6 (which considered discriminant models, the Kalman filter and the proportions of total traffic flows) could be a good alternative to Model 7 if the traffic or geometric conditions were not appropriate for collecting lane-to-lane traffic flows at the target intersection.

The results of the computer simulations and the case study of real-world traffic demonstrated that the proposed method was robust and accurate for the estimation of lane-based queue lengths in real time under a wide range of traffic conditions. Calibrated discriminant models played a significant role in the presence or absence of residual queued vehicles in each lane at the start times of each cycle. In addition, estimating downstream arrivals with the Kalman filter enhanced the accuracy of predictions by minimizing the error terms caused by lane-changing behavior. In future research, these models can be used to establish group-based adaptive control logic in real time, or to adjust the duration, start time and sequence of signal groups by a few seconds or minutes for both isolated signalized junctions and area traffic control. 


\section{Acknowledgements}

The work described in this study was jointly supported by a Research Postgraduate Studentship and grants from the University Research Committee of the University of Hong Kong (201411159005), and the Research Grants Council of the Hong Kong Special Administrative Region, China (Project No. 717512).

\section{References}

Akçelik, R., 1980. Time-dependent expressions for delay, stop rate and queue length at traffic signals. Australian Road Research Board, Melbourne, Australia.

Bishop, G., Welch, G., 2001. An introduction to the Kalman filter. Proc of SIGGRAPH, Course 8, 41.

Chang, G.-L., Su, C.-C., 1995. Predicting intersection queues with neural network models. Transportation Research Part C: Emerging Technologies 3, 175-191.

Chang, J., Talas, M., Muthuswamy, S., 2013. Simple methodology for estimating queue lengths at signalized intersections using detector data. Transportation Research Record: Journal of the Transportation Research Board 2355, 31-38.

Cheng, Y., X. Qin, J. Jin, B. Ran, and J. Anderson, 2011. Cycle by cycle queue length estimation for signalized intersections using sampled trajectory data. In 90th Annual Meeting of the Transportation Research Board. Washington, D.C.

Cheng, Y., X. Qin, J. Jin, and B. Ran, 2012. An exploratory shockwave approach to estimating queue length using probe trajectories. Journal of Intelligent Transportation Systems 16, 12-23.

Christofa, E., Argote, J. and Skabardonis, A., 2013. Arterial Queue Spillback Detection and Signal Control based on Connected Vehicle Technology. Transportation Research Record: Journal of the Transportation Research Board 2356, 61-70.

Chung, E., Lee, J., Qian, G., 2012. Algorithm for queue estimation with loop detector of time occupancy in off-ramps on signalized motorways. Transportation Research Record: Journal of the Transportation Research Board 2278, 50-56.

Comert, G., 2013. Effect of stop line detection in queue length estimation at traffic signals from probe vehicles data. European Journal of Operational Research 226, 67-76.

Comert, G., Cetin, M., 2009. Queue length estimation from probe vehicle location and the impacts of sample size. European Journal of Operational Research 197, 196-202.

Fang, F.C., Elefteriadou, L., 2006. Development of an optimization methodology for adaptive traffic signal control at diamond interchanges. Journal of Transportation Engineering 132, 629-637.

Fu, L., Hellinga, B., Zhu, Y., 2001. An adaptive model for real-time estimation of overflow queues on congested arterials, IEEE Proceedings of Intelligent Transportation Systems, pp. 219-226.

Gartner, N., Pooran, F., Andrews, C., 2002. Optimized policies for adaptive control strategy in realtime traffic adaptive control systems: Implementation and field testing. Transportation Research Record: Journal of the Transportation Research Board 1811, 148-156.

Heidemann, D., 1994. Queue length and delay distributions at traffic signals. Transportation Research Part B: Methodological 28, 377-389.

Heydecker, B.G., Dudgeon, I.W., 1987. Calculation of signal settings to minimise delay at a junction. Proceedings of the 10th International Symposium on Transportation and Traffic Theory. MIT, Elsevier, New York, 159-178.

Kalman, R.E., 1960. A new approach to linear filtering and prediction problems. Journal of Fluids Engineering 82, 35-45.

Lee, J., Jiang, R., Chung, E., 2013. Traffic queue estimation for metered motorway on-ramps through use of loop detector time occupancies. Transportation Research Record: Journal of the Transportation Research Board 2396, 45-53.

Lee, S., Wong, S.C., Pang, C.C.C., Choi, K., 2014. Real-time estimation of lane-to-lane turning flows at isolated signalized junctions. IEEE Transactions on Intelligent Transportation Systems, in press. 
Lighthill, M.J., Whitham, G.B., 1955. On kinematic waves. II. A theory of traffic flow on long crowded roads. Proceedings of the Royal Society of London. Series A. Mathematical and Physical Sciences 229, 317-345.

Li, H., Prevedouros, P.D., 2004. Traffic adaptive control for oversaturated isolated intersections: Model development and simulation testing. Journal of Transportation Engineering 130, 594601.

Li, J.-Q., Zhou, K., Shladover, S.E., Skabardonis, A., 2013. Estimating queue length under connected vehicle technology. Transportation Research Record: Journal of the Transportation Research Board 2356, 17-22.

Lindley, D.V., 1952. The theory of queues with a single server. Mathematical Proceedings of the Cambridge Philosophical Society. Cambridge Univ. Press, pp. 277-289.

Liu, H.X., Wu, X., Ma, W., Hu, H., 2009. Real-time queue length estimation for congested signalized intersections. Transportation Research Part C: Emerging Technologies 17, 412-427.

Liu, H.X., Wu, X., Michalopoulos, P.G., 2007. Improving queue size estimation for Minnesota's stratified zone metering strategy. Transportation Research Record: Journal of the Transportation Research Board 2012, 38-46.

Lu, Y., Chang, G.-L., 2012. Stochastic Model for Estimation of Time-Varying Arterial Travel Time and Its Variability with Only Link Detector Data. Transportation Research Record: Journal of the Transportation Research Board 2283, 44-56.

Lu, Y., Yang, X., 2014. Estimating Dynamic Queue Distribution in a Signalized Network Through a Probability Generating Model. Intelligent Transportation Systems, IEEE Transactions on 15, 334-344.

Mirchandani, P., Head, L., 2001. A real-time traffic signal control system: Architecture, algorithms, and analysis. Transportation Research Part C: Emerging Technologies 9, 415-432.

Mucsi, K., Khan, A.M., Ahmadi, M., 2011. An adaptive neuro-fuzzy inference system for estimating the number of vehicles for queue management at signalized intersections. Transportation Research Part C: Emerging Technologies 19, 1033-1047.

Mung, G.K., Poon, A.C., Lam, W.H., 1996. Distributions of queue lengths at fixed time traffic signals. Transportation Research Part B: Methodological 30, 421-439.

Mystkowski, C., Khan, S., 1999. Estimating queue lengths by using SIGNAL94, SYNCHRO3, TRANSYT-7F, PASSER II-90, and CORSIM. Transportation Research Record: Journal of the Transportation Research Board 1683, 110-117.

NGSIM, 2006. Next generation simulation [WWW Document]. URL: $<$ http://ngsim-community.org/> \{accessed 11.1.14\}

Papamichail, I., Papageorgiou, M., 2011. Balancing of queues or waiting times on metered dualbranch on-ramps, IEEE Transactions on Intelligent Transportation Systems 12, 438-452.

Richards, P.I., 1956. Shock waves on the highway. Operations Research 4, 42-51.

Shelby, S.G., 2004. Single-intersection evaluation of real-time adaptive traffic signal control algorithms. Transportation Research Record: Journal of the Transportation Research Board 1867, 183-192.

Varaiya, P., 2013. Max pressure control of a network of signalized intersections. Transportation Research Part C: Emerging Technologies 36, 177-195.

Vigos, G., Papageorgiou, M., Wang, Y., 2008. Real-time estimation of vehicle-count within signalized links. Transportation Research Part C: Emerging Technologies 16, 18-35.

Vigos, G., Papageorgiou, M., 2010. A simplified estimation scheme for the number of vehicles in signalized links. IEEE Transactions on Intelligent Transportation Systems 11, 312-321.

Viloria, F., Courage, K., Avery, D., 2000. Comparison of queue-length models at signalized intersections. Transportation Research Record: Journal of the Transportation Research Board $1710,222-230$.

Viti, F., van Zuylen, H.J., 2004. Modeling queues at signalized intersections. Transportation Research Record: Journal of the Transportation Research Board 1883, 68-77.

Viti, F., van Zuylen, H.J., 2010a. Probabilistic models for queues at fixed control signals. Transportation Research Part B: Methodological 44, 120-135.

Viti, F., van Zuylen, H.J., 2010b. A probabilistic model for traffic at actuated control signals. Transportation Research Part C: Emerging Technologies 18, 299-310. 
Webster, F., 1958. Traffic signal settings. Road Research Technical Paper no. 39. Road Research Laboratory.

Wong S.C., 1995. Derivatives of performance index for the traffic model from TRANSYT. Transportation Research Part B: Methodological 29, 303-327.

Wong, S.C., 1996. Group-based optimisation of signal timings using the TRANSYT traffic model. Transportation Research Part B: Methodological 30, 217-244.

Wong, C.K., Wong, S.C., 2003. Lane-based optimization of signal timings for isolated junctions. Transportation Research Part B: Methodological 37, 63-84.

Wong, C.K., Wong, S.C., Tong, C.O., 2006. A lane-based optimization method for the multi-period analysis of isolated signal-controlled junctions. Transportmetrica 2, 53-85.

Wu, J., Jin, X., Horowitz, A.J., 2008. Methodologies for estimating vehicle queue length at metered on-ramps. Transportation Research Record: Journal of the Transportation Research Board 2047, 75-82.

Zheng, X., Recker, W., 2013. An adaptive control algorithm for traffic-actuated signals. Transportation Research Part C: Emerging Technologies 30, 93-115. 